\title{
Martingale Morrey-Hardy and Campanato-Hardy Spaces
}

\author{
Eiichi Nakai, ${ }^{1}$ Gaku Sadasue, ${ }^{2}$ and Yoshihiro Sawano ${ }^{3}$ \\ ${ }^{1}$ Department of Mathematics, Ibaraki University, Mito, Ibaraki 310-8512, Japan \\ ${ }^{2}$ Department of Mathematics, Osaka Kyoiku University, Kashiwara, Osaka 582-8582, Japan \\ ${ }^{3}$ Department of Mathematics and Information Sciences, Tokyo Metropolitan University, Minami-Osawa 1-1, \\ Hachioji-shi, Tokyo 192-0397, Japan \\ Correspondence should be addressed to Gaku Sadasue; sadasue@cc.osaka-kyoiku.ac.jp
}

Received 10 June 2013; Accepted 17 August 2013

Academic Editor: Natasha Samko

Copyright (C) 2013 Eiichi Nakai et al. This is an open access article distributed under the Creative Commons Attribution License, which permits unrestricted use, distribution, and reproduction in any medium, provided the original work is properly cited.

We introduce generalized Morrey-Campanato spaces of martingales, which generalize both martingale Lipschitz spaces introduced by Weisz (1990) and martingale Morrey-Campanato spaces introduced in 2012. We also introduce generalized Morrey-Hardy and Campanato-Hardy spaces of martingales and study Burkholder-type equivalence. We give some results on the boundedness of fractional integrals of martingales on these spaces.

\section{Introduction}

Lebesgue spaces $L_{p}$ and Hardy spaces $H_{p}$ play an important role in martingale theory and in harmonic analysis as well. Morrey-Campanato spaces are very useful to know more precise properties of functions and martingales. It is known that Morrey-Campanato spaces contain $L_{p}, \mathrm{BMO}$, and $\operatorname{Lip}_{\alpha}$ as special cases; see, for example, $[1,2]$.

In martingale theory, Weisz [3] introduced martingale Lipschitz spaces for general filtrations $\left\{\mathscr{F}_{n}\right\}_{n \geq 0}$ and proved the duality between martingale Hardy spaces and martingale Lipschitz spaces. This result was extended to generalized martingale Campanato spaces and martingale Orlicz-Hardy spaces in [4]. Recently, martingale Morrey-Campanato spaces were introduced in [5], where each sub- $\sigma$-algebra $\mathscr{F}_{n}$ is generated by countable atoms.

In this paper, we introduce martingale Morrey-Hardy and Campanato-Hardy spaces based on square functions and unify Hardy, Lipschitz, and Morrey-Campanato spaces in [3-5]. To do this, we first introduce generalized martingale Morrey-Campanato spaces by using subfamilies $\left\{\mathscr{B}_{n}\right\}_{n \geq 0}$ of the filtration $\left\{\mathscr{F}_{n}\right\}_{n \geq 0}$ with $\mathscr{B}_{n} \subset \mathscr{F}_{n}$ for each $n \geq 0$. We establish Burkholder-type equivalence and discuss equivalence between martingale Morrey spaces and martingale Campanato spaces in a suitable condition. We also establish a John-Nirenberg-type theorem for generalized martingale Campanato-Hardy spaces; see Theorem 15.
On these martingale spaces, we introduce generalized fractional integrals as martingale transforms and prove their boundedness. Our result extends several results in [5-7] to these spaces. The fractional integrals are very useful tools to analyse function spaces in harmonic analysis. Actually, on the Euclidean space, Hardy and Littlewood [8,9] and Sobolev [10] investigated the fractional integrals to establish the theory of Lebesgue spaces and Lipschitz spaces. Stein and Weiss [11], Taibleson and Weiss [12], and Krantz [13] also investigated the fractional integrals to establish the theory of Hardy spaces; see also [14]. The $L_{p}-L_{q}$ boundedness of the fractional integrals is well known as the Hardy-LittlewoodSobolev theorem derived from [8-10]. This boundedness has been extended to Morrey-Campanato spaces by Peetre [1] and Adams [15]; see also Chiarenza and Frasca [16]. In martingale theory, based on the result on the Walsh multiplier by Watari [7, Theorem 1.1], Chao and Ombe [6] proved the boundedness of the fractional integrals for $H_{p}, L_{p}, \mathrm{BMO}$, and Lipschitz spaces of the dyadic martingale. The boundedness of the fractional integrals for martingale Morrey-Campanato spaces was established in [5]. For other types of operators for martingales, see the recent work by Tanaka and Terasawa [17].

At the end of this section, we make some conventions. Throughout this paper, we always use $C$ to denote a positive constant that is independent of the main parameters involved but whose value may differ from line to line. Constants with 
subscripts, such as $C_{p}$, are dependent on the subscripts. If $f \leq$ $\mathrm{Cg}$, we then write $f \lesssim g$ or $g \gtrsim f$; and if $f \lesssim g \lesssim f$, we then write $f \sim g$.

\section{Definitions and Notation}

Let $(\Omega, \Sigma, P)$ be a probability space and $\mathscr{F}=\left\{\mathscr{F}_{n}\right\}_{n \geq 0}$ a nondecreasing sequence of sub- $\sigma$-algebras of $\Sigma$ such that $\Sigma=$ $\sigma\left(\bigcup_{n} \mathscr{F}_{n}\right)$. For the sake of simplicity, let $\mathscr{F}_{-1}=\mathscr{F}_{0}$. The set $B \in \mathscr{F}_{n}$ is called atom, more precisely $\left(\mathscr{F}_{n}, P\right)$-atom, if any $A \subset B, A \in \mathscr{F}_{n}$, satisfying $P(A)=P(B)$ or $P(A)=0$. Denote by $A\left(\mathscr{F}_{n}\right)$ the set of all atoms in $\mathscr{F}_{n}$.

The expectation operator and the conditional expectation operators relative to $\mathscr{F}_{n}$ are denoted by $E$ and $E_{n}$, respectively. It is known from the Doob theorem that if $p \in(1, \infty)$, then any $L_{p}$-bounded martingale converges in $L_{p}$. Moreover, if $p \in[1, \infty)$, then, for any $f \in L_{p}$, its corresponding martingale $\left(f_{n}\right)_{n \geq 0}$ with $f_{n}=E_{n} f$ is an $L_{p}$-bounded martingale and converges to $f$ in $L_{p}$ (see, e.g., [18]). For this reason a function $f \in L_{1}$ and the corresponding martingale $\left(f_{n}\right)_{n \geq 0}$ will be denoted by the same symbol $f$.

Let $\mathscr{M}$ be the set of all martingales such that $f_{0}=0$. For $p \in[1, \infty]$, let $L_{p}^{0}$ be the set of all $f \in L_{p}$ such that $E_{0} f=0$. For any $f \in L_{p}^{0}$, its corresponding martingale $\left(f_{n}\right)_{n \geq 0}$ with $f_{n}=E_{n} f$ is an $L_{p}$-bounded martingale in $\mathscr{M}$. For this reason, we regard $L_{p}^{0}$ as a subset of $\mathscr{M}$.

Let $\mathscr{B}=\left\{\mathscr{B}_{n}\right\}_{n \geq 0}$ be subfamilies of $\mathscr{F}=\left\{\mathscr{F}_{n}\right\}_{n \geq 0}$ with $\mathscr{B}_{n} \subset \mathscr{F}_{n}$ for each $n \geq 0$. We denote by $\mathscr{B} \subset \mathscr{F}$ this relation of $\mathscr{B}$ and $\mathscr{F}$. on $\mathscr{B}$

In this paper, we always postulate the following condition

There exists a countable subset $\mathscr{B}_{0}^{\prime} \subset \mathscr{B}_{0}$

$$
\text { such that } P\left(\bigcup_{B \in \mathscr{B}_{0}^{\prime}} B\right)=1 \text {. }
$$

We first define generalized martingale Morrey-Campanato spaces with respect to $\mathscr{B}$ as follows.

Definition 1. Let $\mathscr{B} \subset \mathscr{F}, p \in[1, \infty)$, and $\phi:(0,1] \rightarrow$ $(0, \infty)$. For $f \in L_{1}$, let

$$
\begin{aligned}
\|f\|_{L_{p, \phi}} & =\|f\|_{L_{p, \phi}(\mathscr{B})} \\
& =\sup _{n \geq 0} \sup _{B \in \mathscr{B}_{n}} \frac{1}{\phi(P(B))}\left(\frac{1}{P(B)} \int_{B}|f|^{p} d P\right)^{1 / p}, \\
\|f\|_{\mathscr{L}_{p, \phi}} & =\|f\|_{\mathscr{L}_{p, \phi}(\mathscr{B})} \\
& =\sup _{n \geq 0} \sup _{B \in \mathscr{B}_{n}} \frac{1}{\phi(P(B))}\left(\frac{1}{P(B)} \int_{B}\left|f-E_{n} f\right|^{p} d P\right)^{1 / p}, \\
\|f\|_{\mathscr{L}_{p, \phi}^{-}} & =\|f\|_{\mathscr{L}_{p, \phi}^{-}(\mathscr{B})} \\
& =\sup _{n \geq 0} \sup _{B \in \mathscr{B}_{n}} \frac{1}{\phi(P(B))}\left(\frac{1}{P(B)} \int_{B}\left|f-E_{n-1} f\right|^{p} d P\right)^{1 / p},
\end{aligned}
$$

and define

$$
\begin{gathered}
L_{p, \phi}=L_{p, \phi}(\mathscr{B})=\left\{f \in L_{p}^{0}:\|f\|_{L_{p, \phi}}<\infty\right\}, \\
\mathscr{L}_{p, \phi}=\mathscr{L}_{p, \phi}(\mathscr{B})=\left\{f \in L_{p}^{0}:\|f\|_{\mathscr{L}_{p, \phi}}<\infty\right\}, \\
\mathscr{L}_{p, \phi}^{-}=\mathscr{L}_{p, \phi}^{-}(\mathscr{B})=\left\{f \in L_{p}^{0}:\|f\|_{\mathscr{L}_{p, \phi}^{-}}<\infty\right\} .
\end{gathered}
$$

Remark 2. By the condition (1), the functionals $\|f\|_{L_{p, \phi}}$, $\|f\|_{\mathscr{L}_{p, \phi}}$, and $\|f\|_{\mathscr{L}_{p, \phi}^{-}}$are norms on $L_{p}^{0}$.

Remark 3. Let $f \in L_{1}^{0}$. Then, $f \in \mathscr{L}_{p, \phi}$ if and only if its corresponding martingale $\left(f_{n}\right)_{n \geq 0}$ is $\mathscr{L}_{p, \phi}$-bounded; that is, $\sup _{n \geq 0}\left\|f_{n}\right\|_{\mathscr{L}_{p, \phi}}<\infty$. The same conclusion holds for $\mathscr{L}_{p, \phi}^{-}$. Furthermore, if each sub- $\sigma$-algebra $\mathscr{F}_{n}$ is generated by countable atoms, $\mathscr{B}=\left\{A\left(\mathscr{F}_{n}\right)\right\}_{n \geq 0}$ and $\phi$ is almost decreasing, then the same conclusion holds for $L_{p, \phi}$. More precisely, see Proposition 8.

Remark 4. In general, $\|f\|_{\mathscr{L}_{p, \phi}} \leq 2\|f\|_{L_{p, \phi}}$ and hence $L_{p, \phi} \subset$ $\mathscr{L}_{p, \phi}$. Actually, for any $B \in \mathscr{\mathscr { B }}_{n}$,

$$
\begin{aligned}
\left(\int_{B}\left|f-E_{n} f\right|^{p} d P\right)^{1 / p} \leq & \left(\int_{B}|f|^{p} d P\right)^{1 / p} \\
& +\left(\int_{B}\left|E_{n} f\right|^{p} d P\right)^{1 / p} \\
\leq & 2\left(\int_{B}|f|^{p} d P\right)^{1 / p} .
\end{aligned}
$$

Similarly, $\|f\|_{\mathscr{L}_{p, \phi}} \leq 2\|f\|_{\mathscr{L}_{p, \phi}^{-}}$and $\mathscr{L}_{p, \phi}^{-} \subset \mathscr{L}_{p, \phi}$.

Definition 5. For $\phi \equiv 1$, denote $\mathscr{L}_{p, \phi}$ and $\mathscr{L}_{p, \phi}^{-}$by $\mathrm{BMO}_{p}$ and $\mathrm{BMO}_{p}^{-}$, respectively. For $\phi(r)=r^{\alpha}, \alpha>0$, denote $\mathscr{L}_{p, \phi}$ and $\mathscr{L}_{p, \phi}^{-}$by $\operatorname{Lip}_{p, \alpha}$ and $\operatorname{Lip}_{p, \alpha}^{-}$, respectively.

If $\phi(r)=r^{\lambda}, \lambda \in(-\infty, \infty)$, then we simply denote $L_{p, \phi}$, $\mathscr{L}_{p, \phi}$, and $\mathscr{L}_{p, \phi}^{-}$by $L_{p, \lambda}, \mathscr{L}_{p, \lambda}$, and $\mathscr{L}_{p, \lambda}^{-}$, respectively.

A function $\phi:(0,1] \rightarrow(0, \infty)$ is said to be almost increasing (resp., almost decreasing) if there exists a positive constant $C$ such that

$$
\phi(s) \leq C \phi(t) \quad(\text { resp., } \phi(t) \leq C \phi(s)) \quad \text { for } 0<s \leq t \leq 1 .
$$

For the case $\mathscr{B}=\mathscr{F}$, the spaces $\mathrm{BMO}_{p}(\mathscr{F})$ and $\operatorname{Lip}_{p, \alpha}(\mathscr{F})$ with $\alpha \geq 0$, were introduced by Weisz [3].

Recall that $A\left(\mathscr{F}_{n}\right)$ is the set of all atoms in $\mathscr{F}_{n}$ and let $\mathscr{A}=$ $\left\{A\left(\mathscr{F}_{n}\right)\right\}_{n \geq 0}$. Suppose that each sub- $\sigma$-algebra $\mathscr{F}_{n}$ is generated by countable atoms for the time being. Then, $\mathrm{BMO}_{p}(\mathscr{F})=$ $\mathrm{BMO}_{p}(\mathscr{A})$ and $\operatorname{Lip}_{p, \alpha}(\mathscr{F})=\operatorname{Lip}_{p, \alpha}(\mathscr{A})$; see [5]. In general, if $\phi$ is almost increasing, then

$$
\begin{gathered}
L_{p, \phi}(\mathscr{F})=L_{p, \phi}(\mathscr{A}), \\
\mathscr{L}_{p, \phi}(\mathscr{F})=\mathscr{L}_{p, \phi}(\mathscr{A}), \\
\mathscr{L}_{p, \phi}^{-}(\mathscr{F})=\mathscr{L}_{p, \phi}^{-}(\mathscr{A}),
\end{gathered}
$$


with equivalent norms, respectively. However, if $\phi$ is not almost increasing, then these equalities fail in general; see [5].

In this paper, we do not always assume that each sub- $\sigma$ algebra $\mathscr{F}_{n}$ is generated by countable atoms. Let

$$
A\left(\mathscr{F}_{n}\right)^{\perp}=\left\{B \in \mathscr{F}_{n}: P(B \cap A)=0 \forall A \in A\left(\mathscr{F}_{n}\right)\right\},
$$

and let

$$
\mathscr{B}_{n}=A\left(\mathscr{F}_{n}\right) \cup A\left(\mathscr{F}_{n}\right)^{\perp} \quad(n \geq 0) .
$$

In this case, if $\phi$ is almost increasing, then we will show that

$$
\begin{aligned}
L_{p, \phi}(\mathscr{F}) & =L_{p, \phi}(\mathscr{B}), \\
\mathscr{L}_{p, \phi}(\mathscr{F}) & =\mathscr{L}_{p, \phi}(\mathscr{B}), \\
\mathscr{L}_{p, \phi}^{-}(\mathscr{F}) & =\mathscr{L}_{p, \phi}^{-}(\mathscr{B}),
\end{aligned}
$$

with equivalent norms, respectively (see Proposition 9). Moreover, if $\mathscr{F}_{0}$ is nonatomic, then $\mathscr{B}_{n}=\mathscr{F}_{n}$ for all $n \geq 0$. If each sub- $\sigma$-algebra $\mathscr{F}_{n}$ is generated by countable atoms, then $\mathscr{B}_{n}=A\left(\mathscr{F}_{n}\right)$ for all $n \geq 0$. Therefore, our definition generalizes those in [3-5].

Next we define Morrey-Hardy and Campanato-Hardy spaces, based on square functions, with respect to $\mathscr{B}$ as follows. For $f \in \mathscr{M}$, we denote by $S_{n}(f)$ and $S(f)$ the square function of $f$ :

$$
S_{n}(f)=\left(\sum_{k=0}^{n}\left|d_{k} f\right|^{2}\right)^{1 / 2}, \quad S(f)=\left(\sum_{k=0}^{\infty}\left|d_{k} f\right|^{2}\right)^{1 / 2},
$$

where $d_{k} f=f_{k}-f_{k-1}\left(n \geq 0\right.$, with convention $d_{0} f=0$ and $\left.S_{-1}(f)=0\right)$. We further define

$$
S^{(n)}(f)=\left(S(f)^{2}-S_{n}(f)^{2}\right)^{1 / 2}=\left(\sum_{k=n+1}^{\infty}\left|d_{k} f\right|^{2}\right)^{1 / 2} .
$$

Definition 6. Let $\mathscr{B} \subset \mathscr{F}, p \in(0, \infty)$, and $\phi:(0,1] \rightarrow$ $(0, \infty)$. For $f=\left(f_{n}\right)_{n \geq 0} \in \mathscr{M}$, let

$$
\begin{aligned}
\|f\|_{H_{p, \phi}^{S}} & =\|f\|_{H_{p, \phi}^{S}(\mathscr{B})} \\
& =\sup _{n \geq 0} \sup _{B \in \mathscr{B}_{n}} \frac{1}{\phi(P(B))}\left(\frac{1}{P(B)} \int_{B} S(f)^{p} d P\right)^{1 / p}, \\
\|f\|_{\mathscr{H}_{p, \phi}^{S}} & =\|f\|_{\mathscr{H}_{p, \phi}^{S}(\mathscr{B})} \\
& =\sup _{n \geq 0} \sup _{B \in \mathscr{B}_{n}} \frac{1}{\phi(P(B))}\left(\frac{1}{P(B)} \int_{B} S^{(n)}(f)^{p} d P\right)^{1 / p}, \\
\|f\|_{\mathscr{H}_{p, \phi}^{S-}} & =\|f\|_{\mathscr{H}_{p, \phi}^{S-}(\mathscr{B})} \\
& =\sup _{n \geq 0} \sup _{B \in \mathscr{B}_{n}} \frac{1}{\phi(P(B))}\left(\frac{1}{P(B)} \int_{B} S^{(n-1)}(f)^{p} d P\right)^{1 / p},
\end{aligned}
$$

and define

$$
\begin{aligned}
& H_{p, \phi}^{S}=H_{p, \phi}^{S}(\mathscr{B})=\left\{f \in \mathscr{M}:\|f\|_{H_{p, \phi}^{S}}<\infty\right\}, \\
& \mathscr{H}_{p, \phi}^{S}=\mathscr{H}_{p, \phi}^{S}(\mathscr{B})=\left\{f \in \mathscr{M}:\|f\|_{\mathscr{H}_{p, \phi}^{S}}<\infty\right\}, \\
& \mathscr{H}_{p, \phi}^{S-}=\mathscr{H}_{p, \phi}^{S-}(\mathscr{B})=\left\{f \in \mathscr{M}:\|f\|_{\mathscr{H}_{p, \phi}^{S-}}<\infty\right\} .
\end{aligned}
$$

By (1), the functionals $\|f\|_{H_{p, \phi}^{S}},\|f\|_{\mathscr{H}_{p, \phi}^{S}}$, and $\|f\|_{\mathscr{H}_{p, \phi}^{S-}}$ are quasinorms on $\mathscr{M}$.

Remark 7. If we take $\phi \equiv 1$ and $\mathscr{B}=\mathscr{F}$, then the norm $\|f\|_{\mathscr{H}_{p, \phi}^{s-}}$ coincides with the norm $\|f\|_{\mathrm{BMO}_{p}^{S}}$ in $[19$, Definition 2.45]. In this point, our notation is different from the one in [19].

In the end of this section, we present the definition of regularity on $\mathscr{F}$ and the doubling condition on $\phi$. The filtration $\mathscr{F}=\left\{\mathscr{F}_{n}\right\}_{n \geq 0}$ is said to be regular, if there exists a constant $R \geq 2$ such that

$$
f_{n} \leq R f_{n-1}
$$

holds for all nonnegative martingales $\left(f_{n}\right)_{n \geq 0}$. We say the smallest constant $R$ satisfying (14) the regularity constant of $\mathscr{F}$. A function $\phi:(0,1] \rightarrow(0, \infty)$ is said to satisfy the doubling condition if there exists a positive constant $C_{\phi}$ such that

$$
C_{\phi}^{-1} \leq \frac{\phi(s)}{\phi(t)} \leq C_{\phi} \quad \forall s, t \in(0,1] \text { with } \frac{1}{2} \leq \frac{s}{t} \leq 2 .
$$

The smallest constant $C_{\phi}$ satisfying (15) is called the doubling constant of $\phi$.

\section{Properties of Morrey-Hardy and Campanato-Hardy Spaces}

In this section, we investigate the properties of Morrey-Hardy and Campanato-Hardy spaces. The proofs of the results in this section will be given in Section 6 .

First we state basic properties of the norms.

Proposition 8. Let $\mathscr{B} \subset \mathscr{F}, p \in[1, \infty)$ and $\phi:(0,1] \rightarrow$ $(0, \infty)$. Let $f \in L_{1}$ and let $\left(f_{n}\right)_{n \geq 0}$ be its corresponding martingale; $f_{n}=E_{n} f$. Then

$$
\begin{aligned}
& \|f\|_{L_{p, \phi}} \leq \sup _{n \geq 0}\left\|f_{n}\right\|_{L_{p, \phi}}, \\
& \|f\|_{\mathscr{L}_{p, \phi}}=\sup _{n \geq 0}\left\|f_{n}\right\|_{\mathscr{L}_{p, \phi}}, \\
& \|f\|_{\mathscr{L}_{p, \phi}^{-}}=\sup _{n \geq 0}\left\|f_{n}\right\|_{\mathscr{L}_{p, \phi}^{-}} .
\end{aligned}
$$

Moreover, if each sub- $\sigma$-algebra $\mathscr{F}_{n}$ is generated by countable atoms, $\mathscr{B}=\mathscr{A}$, and $\phi$ is almost decreasing; that is, there exists a positive constant $C_{0}$, such that $\phi(t) \leq C_{0} \phi(s)$ for $0<s \leq t \leq 1$, then

$$
\|f\|_{L_{p, \phi}} \leq \sup _{n \geq 0}\left\|f_{n}\right\|_{L_{p, \phi}} \leq C_{0}\|f\|_{L_{p, \phi}}
$$


Proposition 9. Let $A\left(\mathscr{F}_{n}\right) \cup A\left(\mathscr{F}_{n}\right)^{\perp} \subset \mathscr{B}_{n} \subset \mathscr{F}_{n}(n \geq 0)$. If $\phi$ is almost increasing; that is, there exists a positive constant $C_{0}$, such that $\phi(s) \leq C_{0} \phi(t)$ for $0<s \leq t \leq 1$, then

$$
\|f\|_{L_{p, \phi}(\mathscr{B})} \leq\|f\|_{L_{p, \phi}(\mathscr{F})} \leq C_{0}\|f\|_{L_{p, \phi}(\mathscr{B})}
$$

and the same conclusions hold for $\|\cdot\|_{\mathscr{L}_{p, \phi}},\|\cdot\|_{\mathscr{L}_{p, \phi}^{-}},\|\cdot\|_{H_{p, \phi}^{S}}$, $\|\cdot\|_{\mathscr{H}_{p, \phi}^{s}}$, and $\|\cdot\|_{\mathscr{H}_{p, \phi}^{s_{-}}}$. Consequently,

$$
\begin{array}{rlrl}
L_{p, \phi}(\mathscr{F}) & =L_{p, \phi}(\mathscr{B}), & \mathscr{L}_{p, \phi}(\mathscr{F})=\mathscr{L}_{p, \phi}(\mathscr{B}), \\
\mathscr{L}_{p, \phi}^{-}(\mathscr{F})=\mathscr{L}_{p, \phi}^{-}(\mathscr{B}), & H_{p, \phi}(\mathscr{F})=H_{p, \phi}(\mathscr{B}), \\
\mathscr{H}_{p, \phi}^{S}(\mathscr{F})=\mathscr{H}_{p, \phi}^{S}(\mathscr{B}), & \mathscr{H}_{p, \phi}^{S-}(\mathscr{F})=\mathscr{H}_{p, \phi}^{S-}(\mathscr{B}),
\end{array}
$$

with equivalent norms, respectively.

For $p \in(0, \infty)$, let $H_{p}^{S}$ be the set of all $f \in \mathscr{M}$ such that $\|S(f)\|_{L_{p}}<\infty$. Let $\|f\|_{H_{p}^{s}}=\|S(f)\|_{L_{p}}$. Note that if $\phi(r)=$ $r^{-1 / p}$ and $\Omega \in \mathscr{B}_{0}$, then $H_{p, \phi}^{S}=H_{p}^{S}$ and $\|f\|_{H_{p, \phi}^{S}}=\|f\|_{H_{p}^{S}}$.

The following is well known as Burkholder's inequality.

Theorem 10 (Burkholder [20]). If $p \in(1, \infty)$, then there exist positive constants $c_{p}$ and $C_{p}$, that depend only on $p$, such that

$$
c_{p}\|f\|_{L_{p}} \leq\|f\|_{H_{p}^{s}} \leq C_{p}\|f\|_{L_{p}}
$$

for all $f \in L_{1}^{0} \subset \mathscr{M}$.

For expressions of the constants $c_{p}$ and $C_{p}$, see, for example, [21-23]. See also [24] for Burkholder's inequality on Banach functions spaces.

Our first result is the following, which is an extension of Burkholder's inequality to martingale Campanato spaces.

Theorem 11. Let $\mathscr{B} \subset \mathscr{F}, p \in(1, \infty)$ and $\phi:(0,1] \rightarrow$ $(0, \infty)$. Then

$$
\begin{gathered}
c_{p}\|f\|_{\mathscr{L}_{p, \phi}} \leq\|f\|_{\mathscr{H}_{p, \phi}^{S}} \leq C_{p}\|f\|_{\mathscr{L}_{p, \phi}} \\
\frac{c_{p}}{1+c_{p}}\|f\|_{\mathscr{L}_{p, \phi}^{-}} \leq\|f\|_{\mathscr{H}_{p, \phi}^{S^{-}}} \leq\left(2 C_{p}+1\right)\|f\|_{\mathscr{L}_{p, \phi}^{-}}
\end{gathered}
$$

for all $f \in L_{1}^{0} \subset \mathscr{M}$, where $c_{p}$ and $C_{p}$ are the constants in Theorem 10.

Next we give the relations between $\mathscr{L}_{p, \phi}$ and $\mathscr{L}_{p, \phi}^{-}$and between $\mathscr{H}_{p, \phi}^{S}$ and $\mathscr{H}_{p, \phi}^{S-}$. We consider the following condition on $\mathscr{B}$ :

$$
\left\{\omega \in \Omega: E_{n-1}\left[\chi_{B}\right](\omega)>0\right\} \in \mathscr{B}_{n-1} \quad \forall B \in \mathscr{B}_{n}(n \geq 1) .
$$

Theorem 12. Let $\mathscr{B} \subset \mathscr{F}$ and $\phi:(0,1] \rightarrow(0, \infty)$. Then

$$
\begin{aligned}
& \mathscr{L}_{p, \phi} \supset \mathscr{L}_{p, \phi}^{-} \text {with }\|f\|_{\mathscr{L}_{p, \phi}} \leq 2\|f\|_{\mathscr{L}_{p, \phi}^{-}} \text {for } p \in[1, \infty), \\
& \mathscr{H}_{p, \phi}^{S} \supset \mathscr{H}_{p, \phi}^{S-} \text { with }\|f\|_{\mathscr{H}_{p, \phi}^{S}} \leq\|f\|_{\mathscr{H}_{p, \phi}^{S-}} \text { for } p \in(0, \infty) .
\end{aligned}
$$

Conversely, if $\mathscr{F}$ is regular, $\mathscr{B}$ satisfies (23), and $\phi$ satisfies the doubling condition, then there exists a positive constant $C$, dependent only on $p$, the regularity constant of $\mathscr{F}$, and the doubling constant of $\phi$, such that

$$
\mathscr{L}_{p, \phi} \subset \mathscr{L}_{p, \phi}^{-} \quad \text { with } C\|f\|_{\mathscr{L}_{p, \phi}} \geq\|f\|_{\mathscr{L}_{p, \phi}^{-}} \text {for } p \in[1, \infty) \text {, }
$$

$\mathscr{H}_{p, \phi}^{S} \subset \mathscr{H}_{p, \phi}^{S-}$ with $C\|f\|_{\mathscr{H}_{p, \phi}^{S}} \geq\|f\|_{\mathscr{H}_{p, \phi}^{S-}}$ for $p \in(0, \infty)$.

We give a relation between martingale Morrey spaces and martingale Campanato spaces in the following form.

Theorem 13. Suppose that every $\sigma$-algebra $\mathscr{F}_{n}$ is generated by countable atoms. Let $\mathscr{B}=\mathscr{A}, p \in[1, \infty)$, and $\phi:(0,1] \rightarrow$ $(0, \infty)$. Assume that $\phi$ satisfies the doubling condition and there exists a positive constant $C_{\phi}^{\prime}$ such that

$$
\int_{r}^{1} \frac{\phi(t)}{t} d t \leq C_{\phi}^{\prime} \phi(r) \quad(0<r<1) .
$$

Then, there exists a positive constant $C$ such that

$$
\begin{gathered}
\frac{1}{2}\|f\|_{\mathscr{L}_{p, \phi}} \leq\|f\|_{L_{p, \phi}} \leq C\|f\|_{\mathscr{L}_{p, \phi}^{-}} \quad \forall f \in L_{1}^{0}, \\
\|f\|_{\mathscr{H}_{p, \phi}^{S-}} \leq\|f\|_{H_{p, \phi}^{S}} \leq C\|f\|_{\mathscr{H}_{p, \phi}^{S-}} \quad \forall f \in \mathscr{M} .
\end{gathered}
$$

Moreover, if $\mathscr{F}$ is regular, then $\|f\|_{L_{p, \phi}},\|f\|_{\mathscr{L}_{p, \phi}}$, and $\|f\|_{\mathscr{L}_{p, \phi}^{-}}$are equivalent to each other, and $\|f\|_{H_{p, \phi}^{S}},\|f\|_{\mathscr{H}_{p, \phi}^{S}}$ and $\|f\|_{\mathscr{H}_{p, \phi}^{S-}}$ are equivalent to each other.

Using Theorems 11-13, we have Burkholder-type equivalence for generalized martingale Morrey spaces.

Corollary 14. Suppose that every $\sigma$-algebra $\mathscr{F}_{n}$ is generated by countable atoms. Let $\mathscr{B}=\mathscr{A}, p \in(1, \infty)$, and $\phi:(0,1] \rightarrow$ $(0, \infty)$. Assume that $\phi$ satisfies the doubling condition and there exists a positive constant $C_{\phi}^{\prime}$ such that

$$
\int_{r}^{1} \frac{\phi(t)}{t} d t \leq C_{\phi}^{\prime} \phi(r) \quad(0<r<1) .
$$

If $\mathscr{F}$ is regular, then there exist positive constants $c$ and $C$ such that, for all $f \in L_{1}^{0}$,

$$
c\|f\|_{L_{p, \phi}} \leq\|f\|_{H_{p, \phi}^{S}} \leq C\|f\|_{L_{p, \phi}} .
$$

For the martingale BMO spaces based on square functions, the John-Nirenberg-type equivalence was established by Weisz [25] and [19, Theorem 2.50]. We extend this theorem to the spaces $\mathscr{H}_{p, \phi}^{S}$ and $\mathscr{H}_{p, \phi}^{S_{-}}$.

Theorem 15. Let $A\left(\mathscr{F}_{n}\right) \cup A\left(\mathscr{F}_{n}\right)^{\perp} \subset \mathscr{B}_{n} \subset \mathscr{F}_{n}(n \geq 0)$, $p \in(0, \infty)$ and $\phi:(0,1] \rightarrow(0, \infty)$. Assume that $\phi$ is almost increasing and satisfies the doubling condition. Then, $\|f\|_{\mathscr{H}_{p, \phi}^{s}} \leq$ $C_{p, q, \phi}\|f\|_{\mathscr{H}_{q, \phi}^{s-}}$ for all $q \in(0, \infty)$. If we further assume that $\mathscr{F}^{p, \phi}$ is regular, then $\|f\|_{\mathscr{H}_{p, \phi}^{S}}$ and $\|f\|_{\mathscr{H}_{p, \phi}^{s-}}$ are equivalent to $\|f\|_{\mathscr{H}_{2, \phi}^{s}}$. 


\section{Fractional Integrals}

In this section, we state the results on the boundedness of fractional integrals as martingale transforms. The proofs of the results in this section will be given in Section 7 .

Let $\left(\gamma_{n}\right)_{n \geq 0}$ be a sequence of nonnegative bounded functions adapted to $\mathscr{F}=\left\{\mathscr{F}_{n}\right\}_{n \geq 0}$; that is, $\gamma_{n}$ is $\mathscr{F}_{n}$-measurable for every $n \geq 0$. Let $I_{\gamma}$ be the martingale transform associate to $\left(\gamma_{n}\right)_{n \geq 0}$; that is,

$$
\left(I_{\gamma} f\right)_{n}=\sum_{k=0}^{n} \gamma_{k-1} d_{k} f
$$

with convention $\gamma_{-1} d_{0} f=0$. Note that if $f=\left(f_{n}\right)_{n \geq 0} \in \mathscr{M}$, then $I_{\gamma} f=\left(\left(I_{\gamma} f\right)_{n}\right)_{n \geq 0} \in \mathscr{M}$.

We now define a generalized fractional integral $I_{\rho}$ for martingales as a special case of $I_{\gamma}$ under the assumption that every $\sigma$-algebra $\mathscr{F}_{n}$ is generated by countable atoms. Our definition generalizes the fractional integral for dyadic martingales introduced in $[6,7]$. The idea of $I_{\rho}$ comes from [26].

Suppose that every $\sigma$-algebra $\mathscr{F}_{n}$ is generated by countable atoms. Let $b_{n}$ be an $\mathscr{F}_{n}$-measurable function such that

$$
b_{n}(\omega)=P(B) \quad \text { for a.s. } \omega \in B \text { with } B \in A\left(\mathscr{F}_{n}\right) \text {; }
$$

that is,

$$
b_{n}=\sum_{B \in A\left(\mathscr{F}_{n}\right)} P(B) \chi_{B} \quad \text { a.s. }
$$

For a bounded function $\rho:(0,1] \rightarrow(0, \infty)$, we define a generalized fractional integral $I_{\rho} f=\left(\left(I_{\rho} f\right)_{n}\right)_{n \geq 0}$ of $f=\left(f_{n}\right)_{n \geq 0} \in$ $\mathscr{M}$ by

$$
\left(I_{\rho} f\right)_{n}=\sum_{k=0}^{n} \rho\left(b_{k-1}\right) d_{k} f .
$$

The generalized fractional integral $I_{\rho}$ is obtained by taking $\gamma_{n}=\rho\left(b_{n}\right)$ in (32). If $\rho(r)=r^{\alpha}, \alpha>0$, then we simply denote $I_{\rho}$ by $I_{\alpha}$.

For quasinormed spaces $M_{1}$ and $M_{2}$ of martingales, we denote by $B\left(M_{1}, M_{2}\right)$ the set of all bounded martingale transforms from $M_{1}$ to $M_{2}$; that is, $T \in B\left(M_{1}, M_{2}\right)$ means that there exists a positive constant $C$ such that

$$
\|T f\|_{M_{2}} \leq C\|f\|_{M_{1}}
$$

for all martingales $f=\left(f_{n}\right)_{n \geq 0} \in M_{1}$.

We first study the boundedness on the spaces $\mathscr{H}_{p, \phi}^{S}$. On martingale Campanato-Hardy spaces, we consider the fractional integral as a martingale transform associated with monotone multipliers. We say a sequence of nonnegative measurable functions $\gamma=\left(\gamma_{n}\right)_{n \geq 0}$ is almost decreasing if there exists a positive constant $C$ such that

$$
\gamma_{k}(\omega) \leq C \gamma_{\ell}(\omega) \text { a.s. } \forall k \geq \ell .
$$

For an almost decreasing sequence $\gamma=\left(\gamma_{n}\right)_{n \geq 0}$, we define $A_{\gamma}$ by

$$
A_{\gamma}=\inf \{C>0: C \text { satisfies (37) }\} \text {. }
$$

In Theorem 16 below, we do not need any assumption on $\left\{\mathscr{F}_{n}\right\}_{n \geq 0}$.
Theorem 16. Let $\mathscr{B} \subset \mathscr{F}, p \in(0, \infty)$, and $\phi, \psi:(0,1] \rightarrow$ $(0, \infty)$. Let $\left(\gamma_{n}\right)_{n \geq 0}$ be a sequence of nonnegative bounded almost decreasing adapted functions, and let $I_{\gamma}$ be the martingale transform defined by (32). Assume that

$$
C_{\gamma, \phi, \psi}=\sup _{n \geq 0} \sup _{B \in \mathscr{B}_{n}} \frac{\phi(P(B))}{\psi(P(B))}\left\|\gamma_{n} \chi_{B}\right\|_{L_{\infty}}<\infty .
$$

Then

$$
I_{\gamma} \in B\left(\mathscr{H}_{p, \phi}^{S}, \mathscr{H}_{p, \psi}^{S}\right)
$$

with

$$
\left\|I_{\gamma} f\right\|_{\mathscr{H}_{p, \psi}^{S}} \leq A_{\gamma} C_{\gamma, \phi, \psi}\|f\|_{\mathscr{H}_{p, \phi}^{S}}
$$

If every $\sigma$-algebra $\mathscr{F}_{n}$ is generated by countable atoms, then we can apply Theorem 16 to the generalized fractional integral $I_{\rho}$. The following corollary extends [5, Theorem 5.8] to the spaces $\mathscr{H}_{p, \phi}^{S}$.

Corollary 17. Assume that every $\sigma$-algebra $\mathscr{F}_{n}$ is generated by countable atoms and $\mathscr{B}=\mathscr{A}$. Let $p \in(0, \infty)$ and $\rho, \phi, \psi$ : $(0,1] \rightarrow(0, \infty)$. Suppose that $\rho$ is almost increasing and that

$$
\sup _{0<t \leq 1} \frac{\rho(t) \phi(t)}{\psi(t)}<\infty \text {. }
$$

Then

$$
I_{\rho} \in B\left(\mathscr{H}_{p, \phi}^{S}, \mathscr{H}_{p, \psi}^{S}\right)
$$

If one further assumes that $\left\{\mathscr{F}_{n}\right\}_{n \geq 0}$ is regular and that $\psi$ is almost increasing and satisfies the doubling condition, then

$$
I_{\rho} \in B\left(\mathscr{H}_{p, \phi}^{S}, \mathscr{H}_{q, \psi}^{S}\right), \quad(p, q \in(0, \infty)) .
$$

We next study the boundedness on martingale MorreyHardy spaces $H_{p, \phi}^{S}$ and martingale Hardy spaces $H_{p}^{S}$.

Recall that $A\left(\mathscr{F}_{n}\right)^{\perp}=\mathscr{F}_{n}$ for all $n \geq 0$ if $\mathscr{F}_{0}$ is nonatomic.

Proposition 18. Let $\mathscr{B} \subset \mathscr{F}, 0<p<q<\infty$, and $\phi$ : $(0,1] \rightarrow(0, \infty)$. Let $\left(\gamma_{n}\right)_{n \geq 0}$ be a sequence of adapted functions. Suppose that $\mathscr{F}_{0}$ is nonatomic and that $\mathscr{B}=\mathscr{F}$. Assume in addition that $\phi$ is almost decreasing, that $t^{1 / p} \phi(t)$ is almost increasing, and that $\lim _{t \rightarrow 0} \phi(t)=\infty$. Then, $I_{\gamma} \notin$ $B\left(H_{p, \phi}^{S}, H_{q, \phi^{p / q}}^{S}\right) \backslash\{0\}$.

According to Proposition 18, to consider the boundedness on $H_{p, \phi}^{S}$ and $H_{p}^{S}$, we suppose that every $\sigma$-algebra $\mathscr{F}_{n}$ is generated by countable atoms and that $\mathscr{B}=\mathscr{A}$.

In this case, if $\phi(r)=r^{-1 / p}$ and $\mathscr{F}_{0}=\{\Omega, \emptyset\}$, then $H_{p, \phi}^{S}$ coincides with $H_{p}^{S}$ and $\|f\|_{H_{p, \phi}^{S}}=\|f\|_{H_{p}^{S}}$. However, if $\mathscr{F}_{0} \neq\{\Omega, \emptyset\}$, then $H_{p, \phi}^{S}$ does not coincide with $H_{p}^{S}$ in general. We do not always assume that $\mathscr{F}_{0}=\{\Omega, \emptyset\}$.

Theorem 19. Suppose that every $\sigma$-algebra $\mathscr{F}_{n}$ is generated by countable atoms, that $\mathscr{B}=\mathscr{A}$, and that $\left\{\mathscr{F}_{n}\right\}_{n \geq 0}$ is regular. Let 
$0<p<q<\infty$ and $\phi:(0,1] \rightarrow(0, \infty)$, and let $\left(\gamma_{n}\right)_{n \geq 0}$ be a sequence of nonnegative bounded adapted functions. Assume that $\phi$ satisfies the doubling condition and that there exists a positive constant $C$ such that

$$
\begin{aligned}
& \sum_{k=0}^{n} \gamma_{k-1} \phi\left(b_{k-1}\right) \chi_{\left\{b_{k} \neq b_{k-1}\right\}}+\phi\left(b_{n}\right) \\
& \quad \times \sum_{k=n+1}^{\infty} \gamma_{k-1} \chi_{\left\{b_{k} \neq b_{k-1}\right\}} \leq C \phi\left(b_{n}\right)^{p / q} \text { a.s. }
\end{aligned}
$$

for all $n \geq 0$, where $b_{k}$ is the measurable function defined by (33). Then

$$
I_{\gamma} \in B\left(H_{p, \phi}^{S}, H_{q, \phi^{p / q}}^{S}\right)
$$

Furthermore, if $\phi(t)=t^{-1 / p}$, then

$$
I_{\gamma} \in B\left(H_{p}^{S}, H_{q}^{S}\right)
$$

As a consequence of Theorem 19, we have the following corollary, which gives an extension of [5, Corollary 5.7] to the spaces $H_{p, \phi}^{S}$ and gives a martingale Morrey-Hardy version of Gunawan [27, Theorem B]:

Corollary 20. Suppose that every $\sigma$-algebra $\mathscr{F}_{n}$ is generated by countable atoms, that $\mathscr{B}=\mathscr{A}$, and that $\left\{\mathscr{F}_{n}\right\}_{n \geq 0}$ is regular. Let $0<p<q<\infty$ and $\rho, \phi:(0,1] \rightarrow(0, \infty)$. Assume that $\rho$ is bounded, that both $\rho$ and $\phi$ satisfy the doubling condition, and that there exists a positive constant $C$ such that

$$
\begin{array}{r}
\phi(r) \int_{0}^{r} \frac{\rho(t)}{t} d t+\int_{r}^{1} \frac{\phi(t) \rho(t)}{t} d t \leq C \phi(r)^{p / q} \\
(0<r<1) .
\end{array}
$$

Then

$$
I_{\rho} \in B\left(H_{p, \phi}^{S}, H_{q, \phi^{p / q}}^{S}\right)
$$

The following extends the results for dyadic martingales in $[6,7]$ and the result for $0<p \leq 1$ in [28].

Corollary 21. Suppose that every $\sigma$-algebra $\mathscr{F}_{n}$ is generated by countable atoms, that $\mathscr{B}=\mathscr{A}$, and that $\left\{\mathscr{F}_{n}\right\}_{n \geq 0}$ is regular. Let $0<p<q<\infty$ and $-1 / p+\alpha=-1 / q$. Then

$$
I_{\alpha} \in B\left(H_{p}^{S}, H_{q}^{S}\right)
$$

\section{Lemmas}

We prepare some lemmas to prove the results in Sections 3 and 4 .

Lemma 1. Let $\mathscr{B}_{n}$ satisfy $A\left(\mathscr{F}_{n}\right) \cup A\left(\mathscr{F}_{n}\right)^{\perp} \subset \mathscr{B}_{n} \subset \mathscr{F}_{n}(n \geq$ $0)$. Suppose that $\phi:(0,1] \rightarrow(0, \infty)$ is almost increasing; that is, $\phi(r) \leq C_{0} \phi(s)$ for all $0<r \leq s \leq 1$. Then, for all nonnegative functions $F$,

$$
\begin{aligned}
& \sup _{B \in \mathscr{F}_{n}} \frac{1}{\phi(P(B))}\left(\frac{1}{P(B)} \int_{B} F d P\right) \\
& \quad \leq C_{0} \sup _{B \in \mathscr{B}_{n}} \frac{1}{\phi(P(B))}\left(\frac{1}{P(B)} \int_{B} F d P\right) .
\end{aligned}
$$

Proof. Let

$$
\mathcal{N}=\sup _{B \in \mathscr{B}_{n}} \frac{1}{\phi(P(B))}\left(\frac{1}{P(B)} \int_{B} F d P\right) .
$$

For any $B \in \mathscr{F}_{n}$, we can choose the sets $B_{j}, j=0,1,2, \ldots$ (finite or infinite) such that

$$
\begin{gathered}
B=\cup_{j} B_{j}, \quad B_{0} \in A\left(\mathscr{F}_{n}\right)^{\perp}, \quad B_{j} \in A\left(\mathscr{F}_{n}\right), \\
j=1,2, \ldots, \\
P(B)=\sum_{j} P\left(B_{j}\right) .
\end{gathered}
$$

In this case, $B_{j} \in \mathscr{B}_{n}, j=0,1,2, \ldots$, since $A\left(\mathscr{F}_{n}\right) \cup A\left(\mathscr{F}_{n}\right)^{\perp} \subset$ $\mathscr{B}_{n}$. Then

$$
\begin{aligned}
& \frac{1}{\phi(P(B))}\left(\frac{1}{P(B)} \int_{B} F d P\right) \\
& \quad=\frac{1}{\phi(P(B))} \frac{1}{P(B)} \sum_{j}\left(\int_{B_{j}} F d P\right) \\
& \quad=\sum_{j} \frac{P\left(B_{j}\right)}{P(B)}\left(\frac{1}{\phi(P(B)) P\left(B_{j}\right)} \int_{B_{j}} F d P\right) \\
& \quad \leq \sum_{j} \frac{P\left(B_{j}\right)}{P(B)}\left(\frac{C_{0}}{\phi\left(P\left(B_{j}\right)\right) P\left(B_{j}\right)} \int_{B_{j}} F d P\right) \\
& \quad \leq C_{0} \sum_{j} \frac{P\left(B_{j}\right)}{P(B)} \mathscr{N} \\
& \quad=C_{0} \mathcal{N} .
\end{aligned}
$$

This shows the conclusion.

Lemma 2 (see [5, Lemma 3.3]). Suppose that every $\sigma$-algebra $\mathscr{F}_{n}$ is generated by countable atoms and that $\left\{\mathscr{F}_{n}\right\}_{n \geq 0}$ is regular. Then, every sequence

$$
B_{0} \supset B_{1} \supset \cdots \supset B_{n} \supset \cdots, \quad B_{n} \in A\left(\mathscr{F}_{n}\right),
$$

has the following property: for each $n \geq 1$,

$$
B_{n}=B_{n-1} \quad \text { or } \quad\left(1+\frac{1}{R}\right) P\left(B_{n}\right) \leq P\left(B_{n-1}\right) \leq R P\left(B_{n}\right),
$$

where $R$ is the constant in (14). 
Lemma 3. Suppose that every $\sigma$-algebra $\mathscr{F}_{n}$ is generated by countable atoms and that $\left\{\mathscr{F}_{n}\right\}_{n \geq 0}$ is regular. For $B \in A\left(\mathscr{F}_{m}\right)$, let $B_{j} \in A\left(\mathscr{F}_{j}\right)$ be

$$
B=B_{m} \subset B_{m-1} \subset \cdots \subset B_{0} .
$$

Let $\phi:(0,1] \rightarrow(0, \infty)$. Suppose that $\phi$ satisfies the doubling condition. Then, there exists a positive constant $C$, that depends only on $\phi$ and the regularity constant $R$, such that

$$
\sum_{j=\ell+1}^{m} \phi\left(b_{j}\right) \chi_{\left\{b_{j} \neq b_{j-1}\right\}} \leq C \int_{b_{m}}^{b_{\ell}} \frac{\phi(t)}{t} d t \quad \text { on } B,
$$

where $b_{j}$ is the function defined by (33).

Proof. Let $J=\left\{j: b_{j} \neq b_{j-1}\right\}$. Then, by Lemma 2, we have

$$
\begin{aligned}
& \sum_{j=\ell+1}^{m} \phi\left(b_{j}\right) \chi_{\left\{b_{j} \neq b_{j-1}\right\}} \\
& =\sum_{j \in J, \ell<j \leq m} \phi\left(b_{j}\right) \\
& =\sum_{j \in J, \ell<j \leq m} \frac{1}{\log \left(b_{j-1} / b_{j}\right)} \int_{b_{j}}^{b_{j-1}} \frac{\phi\left(b_{j}\right)}{t} d t \\
& \lesssim \sum_{j \in J, \ell<j \leq m} \int_{b_{j}}^{b_{j-1}} \frac{\phi(t)}{t} d t \\
& =\int_{b_{m}}^{b_{\ell}} \frac{\phi(t)}{t} d t .
\end{aligned}
$$

In Theorem 13, we do not assume that $\left\{\mathscr{F}_{n}\right\}_{n \geq 0}$ is regular. Hence, we need the following lemma.

Lemma 4. Let $\phi:(0,1] \rightarrow(0, \infty)$. Suppose that every $\sigma$ algebra $\mathscr{F}_{n}$ is generated by countable atoms. For $B \in A\left(\mathscr{F}_{n}\right)$, let $B_{j} \in A\left(\mathscr{F}_{j}\right)$ be

$$
B=B_{n} \subset B_{n-1} \subset \cdots \subset B_{0} .
$$

For the sequence $\left\{B_{k}\right\}_{k=0}^{n}$ above, one defines a decreasing sequence of integers $n_{j}=n_{j}\left(\left\{B_{k}\right\}_{k=0}^{n}\right)$ inductively by

$$
\begin{gathered}
n_{1}=\sup \left\{k \in[0, n] \cap \mathbb{Z}: P\left(B_{k}\right) \geq 2 P(B)\right\}, \\
n_{j}=\sup \left\{k \in\left[0, n_{j-1}\right] \cap \mathbb{Z}: P\left(B_{k}\right) \geq 2 P\left(B_{n_{j-1}}\right)\right\} \\
(j \geq 2),
\end{gathered}
$$

where one uses the convention $\sup \emptyset=-1$. One further defines

$$
J=\left\{j: n_{j} \geq 0\right\}, \quad n_{j}^{+}=1+n_{j} .
$$

Suppose that $\phi$ satisfies the doubling condition. Then, there exists a positive constant $C$, that depends only on $\phi$, such that

$$
\sum_{j \in J} \phi\left(b_{n_{j}^{+}}\right) \leq C \int_{b_{n}}^{1} \frac{\phi(t)}{t} d t \quad \text { on } B,
$$

where $b_{j}$ is the function defined by (33).
Note that this lemma is the counterpart to the technique in [29, page 1104 , line 5].

Proof. By the definition of $n_{j}$, if $j \in J$, then

$$
b_{n_{j-1}} \leq b_{n_{j}^{+}}<2 b_{n_{j-1}} \leq b_{n_{j}} \text { on } B
$$

where we use the convention $n_{0}=n$.

Using the doubling condition on $\phi$, we have

$$
\sum_{j \in J} \phi\left(b_{n_{j}^{+}}\right) \lesssim \sum_{j \in J} \int_{b_{n_{j-1}}}^{2 b_{n_{j-1}}} \frac{\phi(t)}{t} d t \leq \int_{b_{n}}^{1} \frac{\phi(t)}{t} d t
$$

because the intervals $\left(b_{n_{j-1}}, 2 b_{n_{j-1}}\right)$ are disjointed by (64).

In the proof of Theorem 19, we need the following estimates for the square function of $I_{\gamma} f$.

Lemma 5. Suppose that every $\sigma$-algebra $\mathscr{F}_{n}$ is generated by countable atoms and that $\left\{\mathscr{F}_{n}\right\}_{n \geq 0}$ is regular. Let $p, q \in(0, \infty)$ with $p<q$. Let $\left(\gamma_{n}\right)_{n \geq 0}$ be a sequence of nonnegative bounded adapted functions. Suppose that $\phi:(0,1] \rightarrow(0, \infty)$ satisfies the doubling condition. Assume that there exists a positive constant $C$ such that

$$
\begin{aligned}
& \sum_{k=0}^{n} \gamma_{k-1} \phi\left(b_{k-1}\right) \chi_{\left\{b_{k} \neq b_{k-1}\right\}}+\phi\left(b_{n}\right) \\
& \quad \times \sum_{k=n+1}^{\infty} \gamma_{k-1} \chi_{\left\{b_{k} \neq b_{k-1}\right\}} \leq C \phi\left(b_{n}\right)^{p / q} \quad \text { a.s. }
\end{aligned}
$$

for all $n \geq 0$, where $b_{k}$ is the measurable function defined by (33). Then, for $f \in \mathscr{M}$ with $\|f\|_{H_{p, \phi}^{s}}=1$,

$$
\begin{gathered}
S_{n}\left(I_{\gamma} f\right) \leq C \phi\left(b_{n-1}\right)^{p / q}, \\
S^{(n)}\left(I_{\gamma} f\right) \leq C \phi\left(b_{n}\right)^{p / q-1} S(f)
\end{gathered}
$$

for all $n \geq 0$, where $C$ is a positive constant independent of $f$.

Proof. Let $f \in \mathscr{M}$ such that $\|f\|_{H_{p, \phi}^{s}}=1$. We first show that

$$
\left|d_{k} f\right| \leq C \phi\left(b_{k-1}\right)
$$

where $C$ is a positive constant that depends only on $\phi$ and the regularity constant $R$. Let $B \in A\left(\mathscr{F}_{k}\right)$. Then, on the set $B$, keeping in mind that

$$
\left|d_{k} f\right|=\left(\frac{1}{P(B)} \int_{B}\left|d_{k} f\right|^{p} d P\right)^{1 / p},
$$

we have

$$
\begin{aligned}
\left|d_{k} f\right| & \leq\left(\frac{1}{P(B)} \int_{B} S(f)^{p} d P\right)^{1 / p} \\
& \leq \phi(P(B))\|f\|_{H_{p, \phi}^{s}} \leq \phi\left(b_{k-1}\right) .
\end{aligned}
$$


We have obtained (68). We now show (67). Using (68) and the assumption (66), we have

$$
\begin{aligned}
S_{n}\left(I_{\gamma} f\right)^{2} & =\sum_{k=0}^{n} \gamma_{k-1}^{2}\left|d_{k} f\right|^{2} \\
& \leq \sum_{k=0}^{n} \gamma_{k-1}^{2} \phi\left(b_{k-1}\right)^{2} \chi_{\left\{b_{k} \neq b_{k-1}\right\}} \\
& \leq\left(\sum_{k=0}^{n} \gamma_{k-1} \phi\left(b_{k-1}\right) \chi_{\left\{b_{k} \neq b_{k-1}\right\}}\right)^{2} \\
& \leq \phi\left(b_{n}\right)^{2 p / q} \leq \phi\left(b_{n-1}\right)^{2 p / q}, \\
S^{(n)}\left(I_{\gamma} f\right)^{2} & =\sum_{k=n+1}^{\infty} \gamma_{k-1}^{2}\left|d_{k} f\right|^{2} \\
& \leq S^{(n)}(f)^{2} \sum_{k=n+1}^{\infty} \gamma_{k-1}^{2} \chi_{\left\{b_{k} \neq b_{k-1}\right\}} \\
& \leq S^{(n)}(f)^{2}\left(\sum_{k=n+1}^{\infty} \gamma_{k-1} \chi_{\left\{b_{k} \neq b_{k-1}\right\}}\right)^{2} \\
& \leq \phi\left(b_{n}\right)^{2 p / q-2} S^{(n)}(f)^{2} .
\end{aligned}
$$

Remark 22. In the course of the proof, the embedding $\ell^{1} \hookrightarrow$ $\ell^{2}$ is used. If one does not use the embedding, then

$$
\begin{aligned}
& \sum_{k=0}^{n} \gamma_{k-1}\left|d_{k} f\right| \lesssim \phi\left(b_{n-1}\right)^{p / q}, \\
& \sum_{k=n+1}^{\infty} \gamma_{k-1}\left|d_{k} f\right| \lesssim \phi\left(b_{n}\right)^{p / q-1} S^{(n)}(f) .
\end{aligned}
$$

\section{Proofs of the Results in Section 3}

In this section, we prove the results in Section 3.

Proposition 8 can be proved in the same way as [5, Proposition 2.2], so we omit the proof. Proposition 9 is a direct consequence of Lemma 1. Then, we will prove Theorems 11, 12 , and 13.

Recall that $S^{(n)}(f)$ is defined by (11).

6.1. Proof of Theorem 11. We first show Theorem 11, Burkholder's inequality on generalized martingale Campanato spaces.

Proof of Theorem 11. Let $f \in L_{1}^{0}$ and $B \in \mathscr{B}_{n}$. Then, $f \chi_{B}-$ $E_{n}\left[f \chi_{B}\right] \in L_{1}^{0} \subset \mathscr{M}$ and

$$
d_{k}\left(f \chi_{B}-E_{n}\left[f \chi_{B}\right]\right)= \begin{cases}0 & \text { if } k \leq n, \\ \left(d_{k} f\right) \chi_{B} & \text { if } k>n .\end{cases}
$$

Therefore, we have $S\left(f \chi_{B}-E_{n}\left[f \chi_{B}\right]\right)=S^{(n)}(f) \chi_{B}$. Hence, using Theorem 10, we have

$$
\begin{aligned}
c_{p}\left(\int_{B}\left|f-E_{n} f\right|^{p} d P\right)^{1 / p} & =c_{p}\left\|f \chi_{B}-E_{n}\left[f \chi_{B}\right]\right\|_{L_{p}} \\
& \leq\left\|S\left(f \chi_{B}-E_{n}\left[f \chi_{B}\right]\right)\right\|_{L_{p}} \\
& =\left(\int_{B} S^{(n)}(f)^{p} d P\right)^{1 / p} \\
& \leq C_{p}\left\|f \chi_{B}-E_{n}\left[f \chi_{B}\right]\right\|_{L_{p}} \\
& =C_{p}\left(\int_{B}\left|f-E_{n} f\right|^{p} d P\right)^{1 / p} .
\end{aligned}
$$

We have obtained (21).

We next show (22). Using (74), we have

$$
\begin{aligned}
\left(\int_{B}\left|f-E_{n-1} f\right|^{p} d P\right)^{1 / p} \leq & \left(\int_{B}\left|f-E_{n} f\right|^{p} d P\right)^{1 / p} \\
& +\left(\int_{B}\left|d_{n} f\right|^{p} d P\right)^{1 / p} \\
\leq & c_{p}^{-1}\left(\int_{B} S^{(n)}(f)^{p} d P\right)^{1 / p} \\
& +\left(\int_{B}\left|d_{n} f\right|^{p} d P\right)^{1 / p} \\
\leq & c_{p}^{-1}\left(\int_{B} S^{(n-1)}(f)^{p} d P\right)^{1 / p} \\
& +\left(\int_{B} S^{(n-1)}(f)^{p} d P\right)^{1 / p} .
\end{aligned}
$$

Therefore,

$$
\frac{c_{p}}{1+c_{p}}\|f\|_{\mathscr{L}_{p, \phi}^{-}} \leq\|f\|_{\mathscr{H}_{p, \phi}^{S-}} .
$$

For the converse part, using the inequality

$$
\left(\int_{B}\left|f-E_{n} f\right|^{p} d P\right)^{1 / p} \leq 2\left(\int_{B}\left|f-E_{n-1} f\right|^{p} d P\right)^{1 / p},
$$

which we have mentioned in Remark 4, we obtain

$$
\begin{aligned}
\left(\int_{B} S^{(n-1)}(f)^{p} d P\right)^{1 / p} \leq & \left(\int_{B} S^{(n)}(f)^{p} d P\right)^{1 / p} \\
& +\left(\int_{B}\left|d_{n} f\right|^{p} d P\right)^{1 / p} \\
\leq & C_{p}\left(\int_{B}\left|f-E_{n} f\right|^{p} d P\right)^{1 / p} \\
& +\left(\int_{B}\left|d_{n} f\right|^{p} d P\right)^{1 / p} \\
\leq & \left(2 C_{p}+1\right)\left(\int_{B}\left|f-E_{n-1} f\right|^{p} d P\right)^{1 / p} .
\end{aligned}
$$


That is,

$$
\|f\|_{\mathscr{L}_{p, \phi}^{-}} \leq\left(2 C_{p}+1\right)\|f\|_{\mathscr{H}_{p, \phi}^{S-}} .
$$

6.2. Proof of Theorem 12. We next show Theorem 12, a relation between $\mathscr{L}_{p, \phi}$ and $\mathscr{L}_{p, \phi}^{-}, \mathscr{H}_{p, \phi}^{S}$, and $\mathscr{H}_{p, \phi}^{S-}$.

Proof of Theorem 12. Inequality (24) was mentioned in Remark 4. Inequality (25) is deduced from the inequality $S(f)^{2}-S_{n}(f)^{2} \leq S(f)^{2}-S_{n-1}(f)^{2}$.

We now show (26). Let $B \in \mathscr{B}_{n}$ and $B^{\prime}=\{\omega \in \Omega$ : $\left.E_{n-1}\left[\chi_{B}\right](\omega)>0\right\}$. Since $B^{\prime} \in \mathscr{F}_{n-1}$, we have

$$
E\left[\chi_{\Omega \backslash B^{\prime}} \chi_{B}\right]=E\left[\chi_{\Omega \backslash B^{\prime}} E_{n-1}\left[\chi_{B}\right]\right]=0
$$

that is, $B \subset B^{\prime}$.

Suppose that $\left\{\mathscr{F}_{n}\right\}_{n \geq 0}$ is regular. To show (26), we first prove

$$
B^{\prime}=\left\{\omega \in \Omega: E_{n-1}\left[\chi_{B}\right](\omega) \geq \frac{1}{R}\right\}
$$

where $R$ is the regularity constant. By the definition of $B^{\prime}$, we have $B^{\prime} \supset\left\{\omega \in \Omega: E_{n-1}\left[\chi_{B}\right](\omega) \geq 1 / R\right\}$. We will show the converse. By the regularity, we have $\chi_{B} \leq R E_{n-1}\left[\chi_{B}\right]$. This implies $B \subset\left\{\omega \in \Omega: E_{n-1}\left[\chi_{B}\right](\omega) \geq 1 / R\right\}$, or equivalently,

$$
\chi_{B} \leq \chi_{\left\{E_{n-1}\left[\chi_{B}\right] \geq 1 / R\right\}}
$$

Operating $E_{n-1}$, we have

$$
E_{n-1}\left[\chi_{B}\right] \leq \chi_{\left\{E_{n-1}\left[\chi_{B}\right] \geq 1 / R\right\}}
$$

We have obtained (81).

From (81), we deduce that

$$
P\left(B^{\prime}\right)=E\left[\chi_{\left\{E_{n-1}\left[\chi_{B}\right] \geq 1 / R\right\}}\right] \leq E\left[R E_{n-1}\left[\chi_{B}\right]\right]=R P(B) .
$$

Hence, using the assumption (23) and the doubling condition on $\phi$ with (84), we have

$$
\begin{aligned}
& \frac{1}{P(B)} \int_{B}\left|f-E_{n-1} f\right|^{p} d P \\
& \quad \leq \frac{1}{P(B)} \int_{B^{\prime}}\left|f-E_{n-1} f\right|^{p} d P \\
& \quad \leq \frac{1}{P\left(B^{\prime}\right)} \int_{B^{\prime}}\left|f-E_{n-1} f\right|^{p} d P \\
& \quad \leq \phi\left(P\left(B^{\prime}\right)\right)^{p}\|f\|_{\mathscr{L}_{p, \phi}}^{p} \\
& \quad \leq \phi(P(B))^{p}\|f\|_{\mathscr{L}_{p, \phi}}^{p}
\end{aligned}
$$

We have obtained (26).

By the same way as above, we have (27). The proof is completed.
6.3. Proof of Theorem 13. We now prove Theorem 13, a relation between martingale Morrey spaces and martingale Campanato spaces.

Proof of Theorem 13. The part $\|f\|_{\mathscr{L}_{p, \phi}} \leq 2\|f\|_{L_{p, \phi}}$ was shown in Remark 4, and the part $\|f\|_{\mathscr{H}_{p, \phi}^{S-}} \leq\|f\|_{H_{p, \phi}^{s}}$ is obvious. We now show the part $\|f\|_{L_{p, \phi}} \leqslant\|f\|_{\mathscr{L}_{p, \phi}^{-}}^{p, \phi}$.

Let $B \in A\left(\mathscr{F}_{n}\right)$. We take $B_{k} \in A\left(\mathscr{F}_{k}\right)$ such that $B=B_{n} \subset$ $B_{n-1} \subset \cdots \subset B_{0}$. Let $n_{j}$ be the decreasing sequence of integers defined in Lemma 4 , with convention $n_{0}=n$. Since $n_{j-1} \geq n_{j}$, the function $E_{n_{j-1}} f-E_{n_{j}} f$ is constant on $B_{n_{j-1}}$. Therefore, on the set $B$, we have

$$
\begin{aligned}
& \left|E_{n_{j-1}} f-E_{n_{j}} f\right| \\
& \quad=\left(\frac{1}{P\left(B_{n_{j-1}}\right)} \int_{B_{n_{j-1}}}\left|E_{n_{j-1}} f-E_{n_{j}} f\right|^{p} d P\right)^{1 / p} \\
& \quad \leq 2^{1 / p}\left(\frac{1}{P\left(B_{n_{j}^{+}}\right)} \int_{B_{n_{j}^{+}}}\left|E_{n_{j-1}} f-E_{n_{j}} f\right|^{p} d P\right)^{1 / p} \\
& \quad \leq 2^{1 / p}\|f\|_{\mathscr{L}_{p, \phi}^{-}} \phi\left(P\left(B_{n_{j}^{+}}\right)\right),
\end{aligned}
$$

where $n_{j}^{+}$is the same as in (62).

Let $J$ be the same as in Lemma 4 and let $m=\max J$. Using Lemma 4 and the assumption (28), we have

$$
\begin{aligned}
\left|E_{n} f-E_{n_{m}} f\right| & \leq \sum_{j \in J}\left|E_{n_{j-1}} f-E_{n_{j}} f\right| \\
& \leq\|f\|_{\mathscr{L}_{p, \phi}^{-}} \sum_{j \in J} \phi\left(P\left(B_{n_{j}^{+}}\right)\right) \\
& \leq\|f\|_{\mathscr{L}_{p, \phi}^{-}} \int_{P(B)}^{1} \frac{\phi(t)}{t} d t \\
& \leq\|f\|_{\mathscr{L}_{p, \phi}^{-}} \phi(P(B)) \text { on } B .
\end{aligned}
$$

For $\left|E_{n_{m}} f\right|$, we may assume that $n_{m}>0$. By the definition of $n_{m}$, we have $P\left(B_{1}\right) \leq P\left(B_{0}\right)<2 P\left(B_{n_{m}}\right) \leq 2 P\left(B_{1}\right) \leq 2 P\left(B_{0}\right)$. Therefore,

$$
\begin{aligned}
\phi\left(P\left(B_{1}\right)\right) & \lesssim \int_{P\left(B_{0}\right) / 2}^{P\left(B_{0}\right)} \frac{\phi(t)}{t} d t \\
& \lesssim \int_{P(B)}^{1} \frac{\phi(t)}{t} d t \lesssim \phi(P(B)) .
\end{aligned}
$$

Hence, on the set $B$, the constant $E_{n_{m}} f$ has the following bound:

$$
\begin{aligned}
\left|E_{n_{m}} f\right| & =\left|E_{n_{m}} f-E_{0} f\right| \\
& =\left(\frac{1}{P\left(B_{n_{m}}\right)} \int_{B_{n_{m}}}\left|E_{n_{m}} f-E_{0} f\right|^{p} d P\right)^{1 / p}
\end{aligned}
$$




$$
\begin{aligned}
& \leq 2^{1 / p}\left(\frac{1}{P\left(B_{1}\right)} \int_{B_{1}}\left|E_{n_{m}} f-E_{0} f\right|^{p} d P\right)^{1 / p} \\
& \leq 2^{1 / p}\|f\|_{\mathscr{L}_{p, \phi}^{-}} \phi\left(P\left(B_{1}\right)\right) \leq\|f\|_{\mathscr{L}_{p, \phi}^{-}} \phi(P(B)) .
\end{aligned}
$$

Combining (87) and (89), we have

$$
\left|E_{n} f\right| \lesssim\|f\|_{\mathscr{L}_{p, \phi}^{-}} \phi(P(B)) \quad \text { on } B \text {. }
$$

Using (24) in Theorem 12, we have

$$
\begin{aligned}
\left(\int_{B}|f|^{p} d P\right)^{1 / p} \leq & \left(\int_{B}\left|f-E_{n} f\right|^{p} d P\right)^{1 / p} \\
& +P(B)^{1 / p}\left|E_{n} f\right| \\
\leq & P(B)^{1 / p} \phi(P(B))\|f\|_{\mathscr{L}_{p, \phi}^{-}} \\
& +P(B)^{1 / p} \phi(P(B))\|f\|_{\mathscr{L}_{p, \phi}^{-}} \\
\sim & P(B)^{1 / p} \phi(P(B))\|f\|_{\mathscr{L}_{p, \phi}^{-}}
\end{aligned}
$$

that is,

$$
\|f\|_{L_{p, \phi}} \lesssim\|f\|_{\mathscr{L}_{p, \phi}^{-}}
$$

We can show $\|f\|_{H_{p, \phi}^{s}} \leqslant\|f\|_{\mathscr{H}_{p, \phi}^{s-}}$ by the same way. Indeed, in (86), we can replace $\left|E_{n_{j-1}} f-E_{n_{j}} f\right|$ and $\|f\|_{\mathscr{L}_{p, \phi}^{-}}$by $\left\{S_{n_{j-1}}(f)^{2}-\right.$ $\left.S_{n_{j}}(f)^{2}\right\}^{1 / 2}$ and $\|f\|_{\mathscr{H}_{p, \phi}^{s-}}$, respectively. The rest is similar and

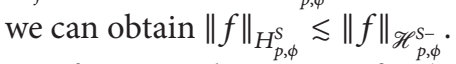

If $\mathscr{B}=\mathscr{A}$, then $\mathscr{B}$ satisfies (23). Therefore, if $\mathscr{F}$ is regular, we can apply Theorem 12 to obtain the equivalence of $\|f\|_{L_{p, \phi}}$, $\|f\|_{\mathscr{L}_{p, \phi}}$, and $\|f\|_{\mathscr{L}_{p, \phi}^{-}}$and the equivalence of $\|f\|_{H_{p, \phi}^{S}},\|f\|_{\mathscr{C}_{p, \phi}^{s}}$, and $\|f\|_{\mathscr{H}_{p, \phi}^{S-}}$.

6.4. Proof of Theorem 15. We will now prove Theorem 15, the John-Nirenberg-type theorem for martingale CampanatoHardy spaces. Following Weisz [19, Definition 2.45], we define

$$
\|f\|_{\mathrm{BMO}_{p}^{S}}=\sup _{n \geq 0}\left\|\left(E_{n}\left[\left\{S(f)^{2}-S_{n-1}(f)^{2}\right\}^{p / 2}\right]\right)^{1 / p}\right\|_{L_{\infty}},
$$

for $f \in \mathscr{M}$ and $p \in(0, \infty)$.

Proof of Theorem 15. We may assume that $\mathscr{B}=\mathscr{F}$ by Proposition 9.

By Hölder's inequality and Theorem 12 , we only need to show that $\|f\|_{\mathscr{H}_{p, \phi}^{S}} \leq C_{p, q, \phi}\|f\|_{\mathscr{H}_{q, \phi}^{S-}}$ for $0<q \leq 1<p$.

Recall the notation $S^{(n)}(f)^{2}=S(f)^{2}-S_{n}(f)^{2}$. Let $f \in$ $\mathscr{H}_{q, \phi}^{S-} \cap L_{1}^{0} \subset \mathscr{M}, A \in \mathscr{F}_{n}$, and $m \geq n+1$. By (73), we have
$S^{(m-1)}\left(f \chi_{A}-E_{n}\left[f \chi_{A}\right]\right)=S^{(m-1)}(f) \chi_{A}$. Hence, for $B \in \mathscr{F}_{m}$, $m \geq n+1$, we have

$$
\begin{aligned}
& \frac{1}{P(B)} \int_{B} S^{(m-1)}\left(f \chi_{A}-E_{n}\left[f \chi_{A}\right]\right)^{q} d P \\
& \quad=\frac{1}{P(B)} \int_{A \cap B} S^{(m-1)}(f)^{q} d P \\
& \quad \leq \frac{1}{P(A \cap B)} \int_{A \cap B} S^{(m-1)}(f)^{q} d P \\
& \leq \phi(P(A \cap B))^{q}\|f\|_{\mathscr{H}_{q, \phi}^{S-}}^{q} \\
& \quad \leq \phi(P(A))^{q}\|f\|_{\mathscr{H}_{q, \phi}^{S-}}^{q} .
\end{aligned}
$$

Therefore, for the $\left\{\mathscr{F}_{m}\right\}_{m \geq n}$-martingale $\left(E_{m}\left[f \chi_{A}\right]-\right.$ $\left.E_{n}\left[f \chi_{A}\right]\right)_{m \geq n}$, we have

$$
\begin{aligned}
& \left\|\left(E_{m}\left[f \chi_{A}\right]-E_{n}\left[f \chi_{A}\right]\right)_{m \geq n}\right\|_{\mathrm{BMO}_{q}^{S}} \\
& \quad \leq \phi(P(A))\|f\|_{\mathscr{H}_{q, \phi}^{S-}} .
\end{aligned}
$$

By [19, Theorem 2.50], there exists a positive constant $C_{p, q, \phi}$ that depends only on $p, q$, and $\phi$ such that

$$
\begin{gathered}
\left(E_{n+1}\left[S^{(n)}\left(f \chi_{A}-E_{n}\left[f \chi_{A}\right]\right)^{p}\right]\right)^{1 / p} \\
\leq C_{p, q, \phi} \phi(P(A))\|f\|_{\mathscr{H}_{q, \phi}^{S-.}}
\end{gathered}
$$

Combining (96) and the fact that $S^{(n)}\left(f \chi_{A}-E_{n}\left[f \chi_{A}\right]\right)=$ $S^{(n)}(f) \chi_{A}$, we have

$$
\left(E_{n+1}\left[S^{(n)}(f)^{p}\right]\right)^{1 / p} \chi_{A} \leq C_{p, q, \phi} \phi(P(A))\|f\|_{\mathscr{H}_{q, \phi}^{S-}} .
$$

Therefore, for $A \in \mathscr{F}_{n}$, we have

$$
\begin{aligned}
& \left(\frac{1}{P(A)} \int_{A} S^{(n)}(f)^{p} d P\right)^{1 / p} \\
& \quad=\left(\frac{1}{P(A)} \int_{A} E_{n+1}\left[S^{(n)}(f)^{p}\right] d P\right)^{1 / p} \\
& \quad \leq C_{p, q, \phi} \phi(P(A))\|f\|_{\mathscr{H}_{q, \phi}^{S-} ;}
\end{aligned}
$$

that is,

$$
\|f\|_{\mathscr{H}_{p, \phi}^{S}} \leq C_{p, q, \phi}\|f\|_{\mathscr{H}_{q, \phi}^{S-}}
$$

for $f \in \mathscr{H}_{q, \phi}^{S_{-}} \cap L_{1}^{0}$. For general $f \in \mathscr{H}_{q, \phi}^{S_{-}}$, applying (99) to the martingale $f^{(m)}=\left(f_{\min (m, n)}\right)_{n \geq 0}$, we have

$$
\left\|f^{(m)}\right\|_{\mathscr{H}_{p, \phi}^{S}} \leq C_{p, q, \phi}\left\|f^{(m)}\right\|_{\mathscr{H}_{q, \phi}^{S-}} \leq C_{p, q, \phi}\|f\|_{\mathscr{H}_{q, \phi}^{S-}} .
$$

Taking $p=2$ in (100), we have that $f$ is an $L^{2}$-bounded martingale. Therefore, we have (99) for all $f \in \mathscr{H}_{q, \phi}^{S_{-}}$. The proof is completed. 


\section{Proofs of the Results in Section 4}

In this section, we prove the results in Section 4.

7.1. Proofs of Theorem 16 and Corollary 17. Recall that $S^{(n)}(f)^{2}=S(f)^{2}-S_{n}(f)^{2}$.

Proof of Theorem 16. Using the assumption that $\left(\gamma_{n}\right)_{n \geq 0}$ is almost decreasing, we have

$$
\begin{aligned}
S^{(n)}\left(I_{\gamma} f\right)^{2} & =\sum_{k=n+1}^{\infty}\left|\gamma_{k-1} d_{k} f\right|^{2} \\
& \leq A_{\gamma}^{2} \gamma_{n}^{2} \sum_{k=n+1}^{\infty}\left|d_{k} f\right|^{2} \\
& =A_{\gamma}^{2} \gamma_{n}^{2} S^{(n)}(f)^{2} .
\end{aligned}
$$

Then, for $B \in \mathscr{B}_{n}$, using the assumption (39), we have

$$
\begin{aligned}
\int_{B} S^{(n)}\left(I_{\gamma} f\right)^{p} d P & \leq A_{\gamma}^{p} \int_{B} \gamma_{n}^{p} S^{(n)}(f)^{p} d P \\
& \leq A_{\gamma}^{p}\left\|\gamma_{n} \chi_{B}\right\|_{L_{\infty}}^{p} \int_{B} S^{(n)}(f)^{p} d P \\
& \leq A_{\gamma}^{p}\left\|\gamma_{n} \chi_{B}\right\|_{L_{\infty}}^{p} P(B) \phi(P(B))^{p}\|f\|_{\mathscr{H}_{p, \phi}^{S}}^{p} \\
& \leq A_{\gamma}^{p} C_{\gamma, \phi, \psi}^{p} P(B) \psi(P(B))^{p}\|f\|_{\mathscr{H} \mathcal{P}_{p, \phi}^{S}}^{p} .
\end{aligned}
$$

Therefore, we have

$$
\left\|I_{\gamma} f\right\|_{\mathscr{H}_{p, \psi}^{S}} \leq A_{\gamma} C_{\gamma, \phi, \psi}\|f\|_{\mathscr{H}_{p, \phi}^{S}}
$$

and $I_{\gamma} \in B\left(\mathscr{H}_{p, \phi}^{S}, \mathscr{H}_{p, \psi}^{S}\right)$. The proof is completed.

Proof of Corollary 17. Let $\gamma_{n}=\rho\left(b_{n}\right)$. Then, we have that $\left(\gamma_{n}\right)_{n \geq 0}$ is almost decreasing and that $\left\|\gamma_{n} \chi_{B}\right\|_{L_{\infty}}=\rho(P(B))$ for $B \in A\left(\mathscr{F}_{n}\right)$. Hence,

$$
\begin{aligned}
C_{\gamma, \phi, \psi} & =\sup _{n \geq 0} \sup _{B \in A\left(\mathscr{F}_{n}\right)} \frac{\rho(P(B)) \phi(P(B))}{\psi(P(B))} \\
& \leq \sup _{0<t \leq 1} \frac{\rho(t) \phi(t)}{\psi(t)}<\infty .
\end{aligned}
$$

Therefore, we can apply Theorem 16 to obtain $I_{\gamma} \in$ $B\left(\mathscr{H}_{p, \phi}^{S}, \mathscr{H}_{p, \psi}^{S}\right)$. If we further assume that $\left\{\mathscr{F}_{n}\right\}_{n \geq 0}$ is regular and that $\psi$ is almost increasing and satisfies the doubling condition, then, by the John-Nirenberg-type equivalence (Theorem 15), we have $I_{\gamma} \in B\left(\mathscr{H}_{p, \phi}^{S}, \mathscr{H}_{q, \psi}^{S}\right)$ for all $q \in$ $(0, \infty)$.
Remark 23. Assume that (39) holds. Suppose further that there exists a positive number $C^{\prime}$ such that $\sum_{k=n+1}^{\infty} \gamma_{k-1} \leq C^{\prime} \gamma_{n}$ a.s. for all $n \geq 0$. Then, in the light of Remark 22, we see that

$$
\begin{aligned}
& \sup _{n \geq 0} \sup _{B \in \mathscr{B}_{n}} \frac{1}{\psi(P(B))}\left(\frac{1}{P(B)} \int_{B}\left(\sum_{k=n+1}^{\infty}\left|\gamma_{k-1} d_{k} f\right|\right)^{p} d P\right)^{1 / p} \\
& \quad \leq C^{\prime} C_{\gamma, \phi, \psi}\|f\|_{\mathscr{H}_{p, \phi}^{S}} .
\end{aligned}
$$

\subsection{Proofs of Theorem 19 and Corollaries 20 and 21}

Proof of Theorem 19. We first show the part $I_{\gamma} \in$ $B\left(H_{p, \phi}^{S}, H_{q, \phi^{p / q}}^{S}\right)$. Assume (45). Let $f=\left(f_{n}\right)_{n \geq 0} \in \mathscr{M}$ such that $\|f\|_{H_{p, \phi}^{s}}=1$. We need only to show that there exists $C>0$ independent of $f$ such that

$$
\left\|I_{\gamma} f\right\|_{H_{q, \phi^{p} / q}^{S}} \leq C
$$

To obtain (106), we first show that

$$
S\left(I_{\gamma} f\right) \leq C S(f)^{p / q}
$$

Let $N=\sum_{n=0}^{\infty} \chi_{\left\{\phi\left(b_{n}\right) \leq S(f)\right\}}$. We define measurable subsets $\Omega_{1}$, $\Omega_{2}$, and $\Omega_{3}$ by

$$
\begin{aligned}
& \Omega_{1}=\{N=\infty\}, \\
& \Omega_{2}=\{N=0\}, \\
& \Omega_{3}=\{0<N<\infty\} .
\end{aligned}
$$

Let $\omega \in \Omega_{1}$. Then, we can take infinitely many integers $n$ such that $\phi\left(b_{n-1}(\omega)\right) \leq S(f)(\omega)$. For such $n$, we have

$$
S_{n}\left(I_{\gamma} f\right)(\omega) \leq C \phi\left(b_{n-1}(\omega)\right)^{p / q} \leq C S(f)(\omega)^{p / q}
$$

by Lemma 5 . Letting $n \rightarrow \infty$ along $n$ that satisfies $\phi\left(b_{n-1}(\omega)\right) \leq S(f)(\omega)$, we have (107) on $\Omega_{1}$.

On $\Omega_{2}$, again by Lemma 5 , we have

$$
S\left(I_{\gamma} f\right) \leq C \phi\left(b_{0}\right)^{p / q-1} S(f) \leq C S(f)^{p / q-1} S(f)=C S(f)^{p / q}
$$

Let $\omega \in \Omega_{3}$. Then, we can take an integer $n$ such that

$$
\phi\left(b_{n-1}(\omega)\right) \leq S(f)(\omega), \quad \phi\left(b_{n}(\omega)\right)>S(f)(\omega) .
$$


Hence, by Lemma 5, we have

$$
\begin{aligned}
S\left(I_{\gamma} f\right)(\omega) \leq & S_{n}\left(I_{\gamma} f\right)(\omega) \\
& +S^{(n)}\left(I_{\gamma} f\right)(\omega) \\
\leq & \phi\left(b_{n-1}(\omega)\right)^{p / q} \\
& +\phi\left(b_{n}(\omega)\right)^{p / q-1} S(f)(\omega) \\
\leq & S(f)(\omega)^{p / q} \\
& +S(f)(\omega)^{p / q-1} S(f)(\omega) \\
\leq & S(f)(\omega)^{p / q} .
\end{aligned}
$$

We have obtained (107). have

We now show (106). Let $B \in \cup_{n} A\left(\mathscr{F}_{n}\right)$. Using (107), we

$$
\begin{aligned}
& \left(\int_{B} S\left(I_{\gamma} f\right)^{q} d P\right)^{1 / q} \\
& \quad \leq\left\{\left(\int_{B} S(f)^{p} d P\right)^{1 / p}\right\}^{p / q} \\
& \quad \leq P(B)^{1 / q} \phi(P(B))^{p / q}\|f\|_{H_{p, \phi}^{s}}^{p / q} \\
& \quad=P(B)^{1 / q} \phi(P(B))^{p / q} .
\end{aligned}
$$

We have obtained (106)

We now show the part $I_{\gamma} \in B\left(H_{p}^{S}, H_{q}^{S}\right)$. Let $\phi(t)=t^{-1 / p}$. We simply denote $H_{p, \phi}^{S}$ by $H_{p,-1 / p}^{S}$. Let $f=\left(f_{n}\right)_{n \geq 0} \in \mathscr{M}$ such that $\|f\|_{H_{p}^{s}}=1$. Observe that $\|f\|_{H_{p,-1 / p}^{s}} \leq\|f\|_{H_{p}^{s}}$. By the assumption that (45) holds for $\phi(t)=t^{-1 / p}$, we can apply (107) to $f /\|f\|_{H_{p,-1 / p}^{s}}$, and we have

$$
S\left(I_{\gamma} f\right) \leq C S(f)^{p / q}\|f\|_{H_{p,-1 / p}^{S}}^{1-p / q} \leq C S(f)^{p / q}
$$

Hence, we obtain

$$
\left(\int_{\Omega} S\left(I_{\gamma} f\right)^{q} d P\right)^{1 / q} \lesssim\left\{\left(\int_{\Omega} S(f)^{p} d P\right)^{1 / p}\right\}^{p / q}=1 .
$$

The proof is completed.

Proof of Corollary 20. Let $\gamma_{n}=\rho\left(b_{n}\right)$. We only have to verify (45). Using Lemma 3 and the assumption (48), we have

$$
\begin{aligned}
& \sum_{k=0}^{n} \rho\left(b_{k-1}\right) \phi\left(b_{k-1}\right) \chi_{\left\{b_{k} \neq b_{k-1}\right\}} \\
& \quad+\phi\left(b_{n}\right) \sum_{k=n+1}^{\infty} \rho\left(b_{k-1}\right) \chi_{\left\{b_{k} \neq b_{k-1}\right\}}
\end{aligned}
$$

$$
\begin{aligned}
& \lesssim \int_{b_{n}}^{1} \frac{\rho(t) \phi(t)}{t} d t \\
& \quad+\phi\left(b_{n}\right) \int_{0}^{b_{n}} \frac{\rho(t)}{t} d t \lesssim \phi\left(b_{n}\right)^{p / q} .
\end{aligned}
$$

By Theorem 19, we have the conclusion.

Proof of Corollary 21. If $\rho(r)=r^{\alpha}$ and $\phi(t)=t^{-1 / p}$, then

$$
\begin{gathered}
\int_{r}^{1} \frac{\rho(t) \phi(t)}{t} d t+\phi(r) \int_{0}^{r} \frac{\rho(t)}{t} d t \sim r^{\alpha-1 / p} \\
=r^{-1 / q}=\phi(r)^{p / q} .
\end{gathered}
$$

Observing (116) and applying Theorem 19 to $I_{\alpha}$, we have the conclusion.

Remark 24. In the light of Remark 22, we see that

$$
\begin{aligned}
& \sup _{n \geq 0} \sup _{B \in A\left(\mathscr{F}_{n}\right)} \frac{1}{\phi(P(B))^{p / q}} \\
& \quad \times\left(\frac{1}{P(B)} \int_{B}\left(\sum_{k=1}^{\infty}\left|\gamma_{k-1} d_{k} f\right|\right)^{q} d P\right)^{1 / q} \\
& \leq C\|f\|_{H_{p, \phi}^{S}},
\end{aligned}
$$

which is similar to (105).

In words of harmonic analysis, this corresponds to the embedding $F_{p_{1} q_{1}}^{s+n / p_{1}-n / p_{2}} \hookrightarrow F_{p_{2} q_{2}}^{s}$ for $0<p_{1}<p_{2}<\infty$, $0<q_{1}, q_{2} \leq \infty$, and $s \in \mathbb{R}$; see [30, Section 2.3] and [30, page 129] for the definition of the space $F_{p q}^{\mathcal{S}}$ and the above embedding, respectively. It may be interesting to observe that this embedding is translated into the fact that $I_{\alpha}$ makes functions have bounded variation.

7.3. Proof of Proposition 18. In this subsection, we prove Proposition 18.

Proof of Proposition 18. To prove $I_{\gamma} \notin B\left(H_{p, \phi}^{S}, H_{q, \phi^{p / q}}^{S}\right) \backslash\{0\}$, we only need to show the following for any $f=\left(f_{n}\right)_{n \geq 0} \in \mathscr{M}$ :

$$
\begin{array}{r}
\text { if } I_{\gamma} \in B\left(H_{p, \phi}^{S}, H_{q, \phi^{p / q}}^{S}\right) \text {, then } \chi_{\left\{\left|\gamma_{k-1}\right|>0\right\}} d_{k} f=0 \\
(k=1,2, \ldots) .
\end{array}
$$

We now show (119) for $f=\left(f_{n}\right)_{n \geq 0} \in \mathscr{M}$. We may assume that $P\left(\left|\gamma_{k-1}\right|>0\right)>0$. For $\gamma_{k-1}$, define

$$
\begin{aligned}
\mathscr{F}_{k}^{+} & \left(\left\{\left|\gamma_{k-1}\right|>0\right\}\right) \\
& =\left\{B \in \mathscr{F}_{k} \backslash \mathscr{F}_{k-1}: P\left(\left\{\left|\gamma_{k-1}\right|>0\right\} \cap B\right)>0\right\} .
\end{aligned}
$$

If $\mathscr{F}_{k}^{+}\left(\left\{\left|\gamma_{k-1}\right|>0\right\}\right)=\emptyset$, then the function $\chi_{\left\{\left|\gamma_{k-1}\right|>0\right\}} d_{k} f$ is $\mathscr{F}_{k-1}$-measurable. Therefore, we have

$$
\begin{aligned}
\chi_{\left\{\left|\gamma_{k-1}\right|>0\right\}} d_{k} f & =E_{k-1}\left[\chi_{\left\{\left|\gamma_{k-1}\right|>0\right\}} d_{k} f\right] \\
& =\chi_{\left\{\left|\gamma_{k-1}\right|>0\right\}} E_{k-1}\left[d_{k} f\right]=0 .
\end{aligned}
$$


To complete the proof of (119), we only have to show the following:

$$
\text { if } \mathscr{F}_{k}^{+}\left(\left\{\left|\gamma_{k-1}\right|>0\right\}\right) \neq \emptyset \text {, then } I_{\gamma} \notin B\left(H_{p, \phi}^{S}, H_{q, \phi^{p / q}}^{S}\right) \text {. }
$$

Assume that $\mathscr{F}_{k}^{+}\left(\left\{\left|\gamma_{k-1}\right|>0\right\}\right) \neq \emptyset$. We fix $B \in \mathscr{F}_{k} \backslash \mathscr{F}_{k-1}$ and $\delta>0$ such that $P\left(\left\{\left|\gamma_{k-1}\right| \geq \delta\right\} \cap B\right)>0$. Let $B_{1}=\left\{\left|\gamma_{k-1}\right| \geq\right.$ $\delta\}$ and $B_{1}^{\prime}=\left\{\left|\gamma_{k-1}\right| \geq \delta\right\} \cap B$. Note that $B_{1} \in \mathscr{F}_{k-1}, B_{1}^{\prime} \in$ $\mathscr{F}_{k}^{+}\left(\left\{\left|\gamma_{k-1}\right|>0\right\}\right)$, and $B_{1}^{\prime} \subset B_{1}$.

To prove (122), we define two decreasing sequences of measurable sets $\left\{B_{n}\right\}_{n=1}^{\infty}$ and $\left\{B_{n}^{\prime}\right\}_{n=1}^{\infty}$ that satisfy

$$
\begin{aligned}
& B_{n} \in \mathscr{F}_{k-1}, \quad P\left(B_{n}\right)=\frac{P\left(B_{1}\right)}{2^{n-1}}, \\
& B_{n}^{\prime} \in \mathscr{F}_{k}^{+}\left(\left\{\left|\gamma_{k-1}\right|>0\right\}\right), \quad B_{n}^{\prime} \subset B_{n}
\end{aligned}
$$

for every $n \geq 1$, inductively as follows.

Suppose that we can choose $B_{n-1}$ and $B_{n-1}^{\prime}$ that satisfy

$$
\begin{aligned}
& B_{n-1} \in \mathscr{F}_{k-1}, \quad P\left(B_{n-1}\right)=\frac{P\left(B_{1}\right)}{2^{n-2}}, \\
& B_{n-1}^{\prime} \in \mathscr{F}_{k}^{+}\left(\left\{\left|\gamma_{k-1}\right|>0\right\}\right), \quad B_{n-1}^{\prime} \subset B_{n-1} .
\end{aligned}
$$

By the assumption that $\mathscr{F}_{0}$ is nonatomic, $\mathscr{F}_{k-1}$ is also nonatomic. Hence, there exists $B_{n} \in \mathscr{F}_{k-1}$ such that $B_{n} \subset$ $B_{n-1}, P\left(B_{n}\right)=P\left(B_{n-1}\right) / 2$ and $P\left(B_{n} \cap B_{n-1}^{\prime}\right)>0$. Let $B_{n}^{\prime}=B_{n} \cap$ $B_{n-1}^{\prime}$. Then, we have $B_{n}$ and $B_{n}^{\prime}$ with $B_{n} \subset B_{n-1}$ and $B_{n}^{\prime} \subset B_{n-1}^{\prime}$ that satisfy (123).

For the set $B_{n}^{\prime}$ defined above, let $g_{n}=\chi_{B_{n}^{\prime}}-E_{k-1}\left[\chi_{B_{n}^{\prime}}\right]$. Since $B_{n}$ is $\mathscr{F}_{k-1}$-measurable, we have

$$
\begin{aligned}
g_{n} \chi_{\Omega \backslash B_{n}} & =\left(\chi_{B_{n}^{\prime}}-E_{k-1}\left[\chi_{B_{n}^{\prime}}\right]\right) \chi_{\Omega \backslash B_{n}} \\
& =\chi_{B_{n}^{\prime}} \chi_{\Omega \backslash B_{n}}-E_{k-1}\left[\chi_{B_{n}^{\prime}} \chi_{\Omega \backslash B_{n}}\right] \\
& =0 .
\end{aligned}
$$

By (125) and the assumption that $t^{1 / p} \phi(t)$ is almost increasing, we have, for any $A \in \cup_{n=0}^{\infty} \mathscr{F}_{n}$,

$$
\begin{aligned}
& \frac{1}{\phi(P(A))}\left(\frac{1}{P(A)} \int_{A}\left|g_{n}\right|^{p} d P\right)^{1 / p} \\
& =\frac{1}{\phi(P(A)) P(A)^{1 / p}}\left(\int_{A \cap B_{n}}\left|g_{n}\right|^{p} d P\right)^{1 / p} \\
& \lesssim \frac{1}{\phi\left(P\left(A \cap B_{n}\right)\right) P\left(A \cap B_{n}\right)^{1 / p}} \\
& \times\left(\int_{A \cap B_{n}}\left|g_{n}\right|^{p} d P\right)^{1 / p} \\
& =\frac{1}{\phi\left(P\left(A \cap B_{n}\right)\right)} \\
& \times\left(\frac{1}{P\left(A \cap B_{n}\right)} \int_{A \cap B_{n}}\left|g_{n}\right|^{p} d P\right)^{1 / p} .
\end{aligned}
$$

We now show that $I_{\gamma} \notin B\left(H_{p, \phi}^{S}, H_{q, \phi^{p / q}}^{S}\right)$. Suppose that $I_{\gamma} \in$ $B\left(H_{p, \phi}^{S}, H_{q, \phi^{p / q}}^{S}\right)$; that is, there exists a positive number $C$ such that

$$
\left\|I_{\gamma} f\right\|_{H_{q, \phi^{p} / q}^{S}} \leq C\|f\|_{H_{p, \phi}^{S}}
$$

for all $f \in H_{p, \phi}^{S}$.

Since $B_{n}^{\prime} \in \mathscr{F}_{k} \backslash \mathscr{F}_{k-1}$, we have $d_{k} g_{n}=g_{n} \neq 0$, and $d_{j} g_{n}=$ 0 for $j \neq k$.

Therefore, we have

$$
S\left(g_{n}\right)=g_{n}, \quad S\left(I_{\gamma} g_{n}\right)=\gamma_{k-1} g_{n} .
$$

For $g_{n}$, we take $D_{n} \in \cup_{n=0}^{\infty} \mathscr{F}_{n}$ such that

$$
\frac{1}{\phi\left(P\left(D_{n}\right)\right)}\left(\frac{1}{P\left(D_{n}\right)} \int_{D_{n}}\left|g_{n}\right|^{p} d P\right)^{1 / p} \geq \frac{1}{2}\left\|g_{n}\right\|_{H_{p, \phi}^{s}} .
$$

By (126), we may assume that $D_{n} \subset B_{n}$. As a consequence, we have $D_{n} \subset B_{1}=\left\{\left|\gamma_{k-1}\right| \geq \delta\right\}$, and $\lim _{n \rightarrow \infty} P\left(D_{n}\right)=0$ by (123). Then, using (127) with (128), we have

$$
\begin{aligned}
& \frac{1}{\phi\left(P\left(D_{n}\right)\right)^{p / q}}\left(\frac{1}{P\left(D_{n}\right)} \int_{D_{n}}\left|g_{n}\right|^{q} d P\right)^{1 / q} \\
& \leq \frac{1}{\delta} \frac{1}{\phi\left(P\left(D_{n}\right)\right)^{p / q}} \\
& \times\left(\frac{1}{P\left(D_{n}\right)} \int_{D_{n}}\left|\gamma_{k-1} g_{n}\right|^{q} d P\right)^{1 / q} \\
& \leq \frac{1}{\delta}\left\|I_{\gamma} g_{n}\right\|_{H_{q, \phi}^{S} / q} \\
& \leq \frac{C}{\delta}\left\|g_{n}\right\|_{H_{p, \phi}^{S}} \\
& \leq \frac{2 C}{\delta} \frac{1}{\phi\left(P\left(D_{n}\right)\right)} \\
& \times\left(\frac{1}{P\left(D_{n}\right)} \int_{D_{n}}\left|g_{n}\right|^{p} d P\right)^{1 / p} \\
& \leq \frac{2 C}{\delta} \frac{1}{\phi\left(P\left(D_{n}\right)\right)} \\
& \times\left(\frac{1}{P\left(D_{n}\right)} \int_{D_{n}}\left|g_{n}\right|^{q} d P\right)^{1 / q} .
\end{aligned}
$$

Therefore, we have

$$
\phi\left(P\left(D_{n}\right)\right)^{1-p / q} \leq \frac{2 C}{\delta} .
$$

However, this contradicts $p<q$ and $\lim _{t \rightarrow 0} \phi(t)=\infty$. We have (122) and hence have (119).

\section{Conflict of Interests}

The authors declare that there is no conflict of interests regarding the publication of this paper. 


\section{Acknowledgments}

The authors are thankful to Professor Masaaki Fukasawa in Osaka University for his kind hint about Remarks 22, 23, and 24. The first author was supported by Grant-in-Aid for Scientific Research (C) (no. 24540159), Japan Society for the Promotion of Science. The second author was supported by Grant-in-Aid for Scientific Research (C) (no. 24540171), Japan Society for the Promotion of Science. The third author was supported by Grant-in-Aid for Young Scientists (B) (no. 24740085), Japan Society for the Promotion of Science.

\section{References}

[1] J. Peetre, "On the theory of $\mathscr{L}_{p, \lambda}$ spaces," Journal of Functional Analysis, vol. 4, no. 1, pp. 71-87, 1969.

[2] W. Yuan, W. Sickel, and D. Yang, Morrey and Campanato Meet Besov, Lizorkin and Triebel, Lecture Notes in Mathematics, Springer, Berlin, Germany, 2010.

[3] F. Weisz, "Martingale Hardy spaces for $0<P \leq 1$," Probability Theory and Related Fields, vol. 84, no. 3, pp. 361-376, 1990.

[4] T. Miyamoto, E. Nakai, and G. Sadasue, "Martingale OrliczHardy spaces," Mathematische Nachrichten, vol. 285, no. 5-6, pp. 670-686, 2012.

[5] E. Nakai and G. Sadasue, "Martingale Morrey-Campanato spaces and fractional integrals," Journal of Function Spaces and Applications, vol. 2012, Article ID 673929, 29 pages, 2012.

[6] J.-A. Chao and H. Ombe, "Commutators on dyadic martingales," Proceedings of the Japan Academy, vol. 61, no. 2, pp. 35-38, 1985.

[7] C. Watari, "Multipliers for Walsh-Fourier series," The Tohoku Mathematical Journal, vol. 16, pp. 239-251, 1964.

[8] G. H. Hardy and J. E. Littlewood, "Some properties of fractional integrals. I," Mathematische Zeitschrift, vol. 27, no. 1, pp. 565606, 1928.

[9] G. H. Hardy and J. E. Littlewood, "Some properties of fractional integrals. II," Mathematische Zeitschrift, vol. 34, no. 1, pp. 403439, 1932.

[10] S. L. Sobolev, "On a theorem in functional analysis," Matematicheskii Sbornik, vol. 4, pp. 471-497, 1938.

[11] E. M. Stein and G. Weiss, "On the theory of harmonic functions of several variables. I. The theory of $H^{p}$-spaces," Acta Mathematica, vol. 103, pp. 25-62, 1960.

[12] M. H. Taibleson and G. Weiss, "The molecular characterization of certain Hardy spaces," in Representation theorems for Hardy spaces, vol. 77 of Astérisque, pp. 67-149, Soc. Math. France, Paris, 1980.

[13] S. G. Krantz, "Fractional integration on Hardy spaces," Studia Mathematica, vol. 73, no. 2, pp. 87-94, 1982.

[14] E. Nakai, "Recent topics of fractional integrals," Sugaku Expositions, vol. 20, no. 2, pp. 215-235, 2007.

[15] D. R. Adams, "A note on Riesz potentials," Duke Mathematical Journal, vol. 42, no. 4, pp. 765-778, 1975.

[16] F. Chiarenza and M. Frasca, "Morrey spaces and HardyLittlewood maximal function," Rendiconti di Matematica e delle sue Applicazioni, vol. 7, no. 3-4, pp. 273-279, 1987.

[17] H. Tanaka and Y. Terasawa, "Positive operators and maximal operators in a filtered measure space," Journal of Functional Analysis, vol. 264, no. 4, pp. 920-946, 2013.
[18] J. Neveu, Discrete-Parameter Martingales, North-Holland, Amsterdam, The Netherlands, 1975.

[19] F. Weisz, Martingale Hardy Spaces and Their Applications in Fourier Analysis, vol. 1568 of Lecture Notes in Mathematics, Springer, Berlin, Germany, 1994.

[20] D. L. Burkholder, "Martingale transforms," Annals of Mathematical Statistics, vol. 37, pp. 1494-1504, 1966.

[21] D. L. Burkholder, "Sharp inequalities for martingales and stochastic integrals, Colloque Paul Lévy sur les Processus Stochastiques (Palaiseau, 1987)," Astérisque, no. 157-158, pp. 7594, 1988.

[22] D. L. Burkholder, "Explorations in martingale theory and its applications," in École d'Été de Probabilités de Saint-Flour XIX 1989, vol. 1464 of Lecture Notes in Mathematics, pp. 1-66, Springer, Berlin, Germany, 1991.

[23] R. L. Long, Martingale Spaces and Inequalities, Peking University Press, Beijing, China, 1993.

[24] M. Kikuchi, "Characterization of Banach function spaces that preserve the Burkholder square-function inequality," Illinois Journal of Mathematics, vol. 47, no. 3, pp. 867-882, 2003.

[25] F. Weisz, "Interpolation between martingale Hardy and BMO spaces, the real method," Bulletin des Sciences Mathématiques, vol. 116, no. 2, pp. 145-158, 1992.

[26] E. Nakai, “On generalized fractional integrals," Taiwanese Journal of Mathematics, vol. 5, no. 3, pp. 587-602, 2001.

[27] H. Gunawan, "A note on the generalized fractional integral operators," Journal of the Indonesian Mathematical Society, vol. 9, no. 1, pp. 39-43, 2003.

[28] G. Sadasue, "Fractional integrals on martingale Hardy spaces for $0<P \leq 1$," Memoirs of Osaka Kyoiku University, vol. 60, no. 1, pp. 1-7, 2011.

[29] Y. Sawano and H. Tanaka, "Sharp maximal inequalities and commutators on Morrey spaces with non-doubling measures," Taiwanese Journal of Mathematics, vol. 11, no. 4, pp. 1091-1112, 2007.

[30] H. Triebel, Theory of Function Spaces, Birkhäuser, Basel, Switzerland, 1983. 


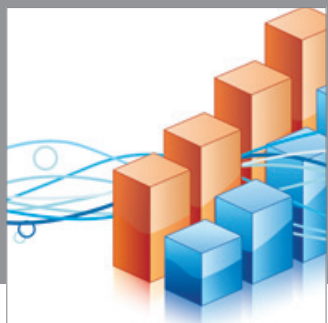

Advances in

Operations Research

mansans

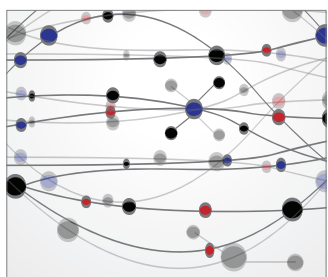

The Scientific World Journal
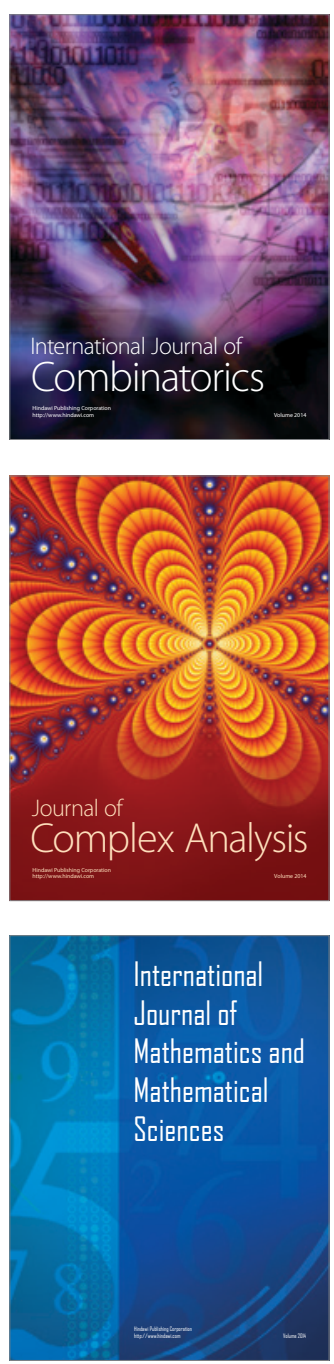
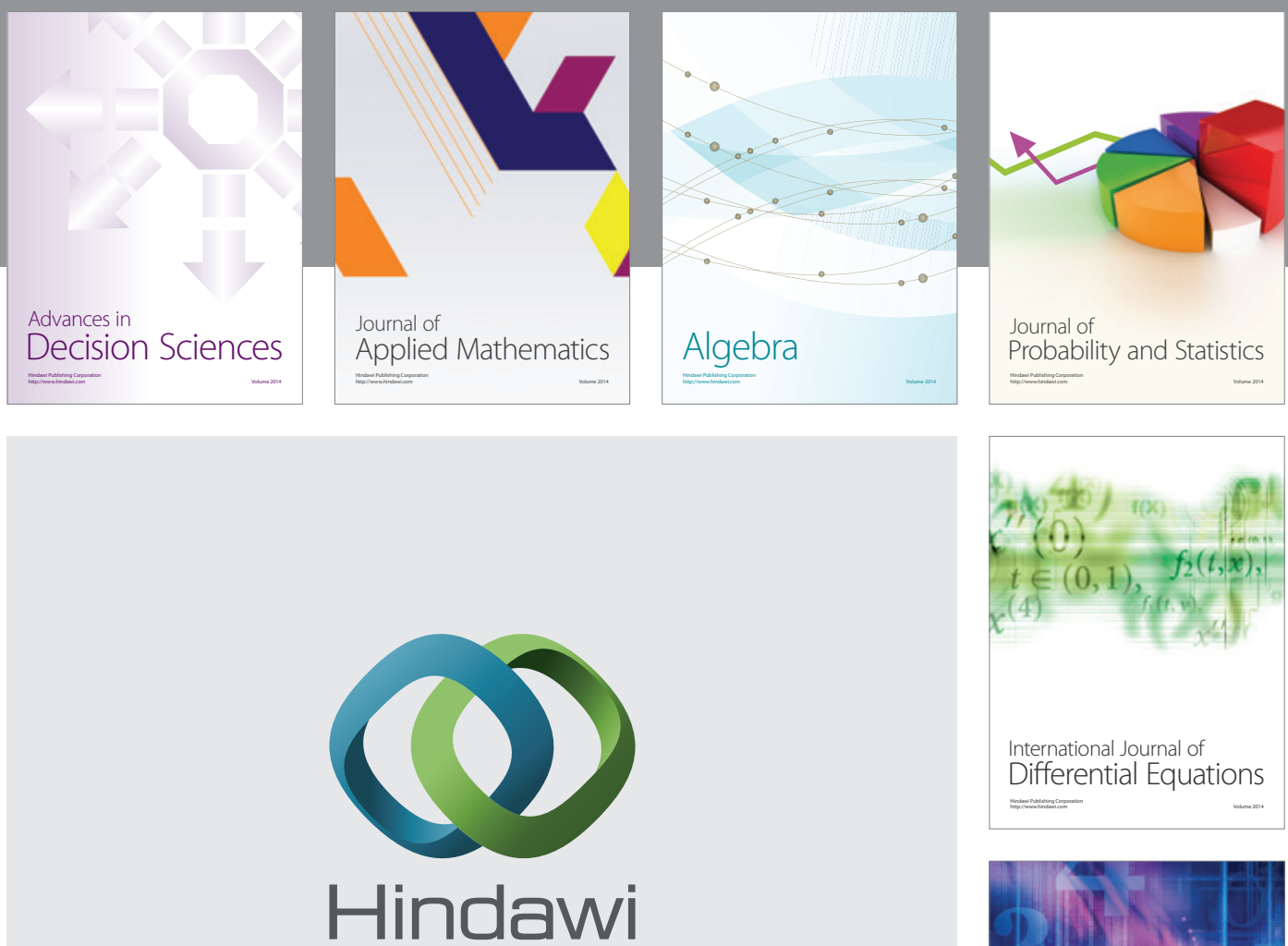

Submit your manuscripts at http://www.hindawi.com
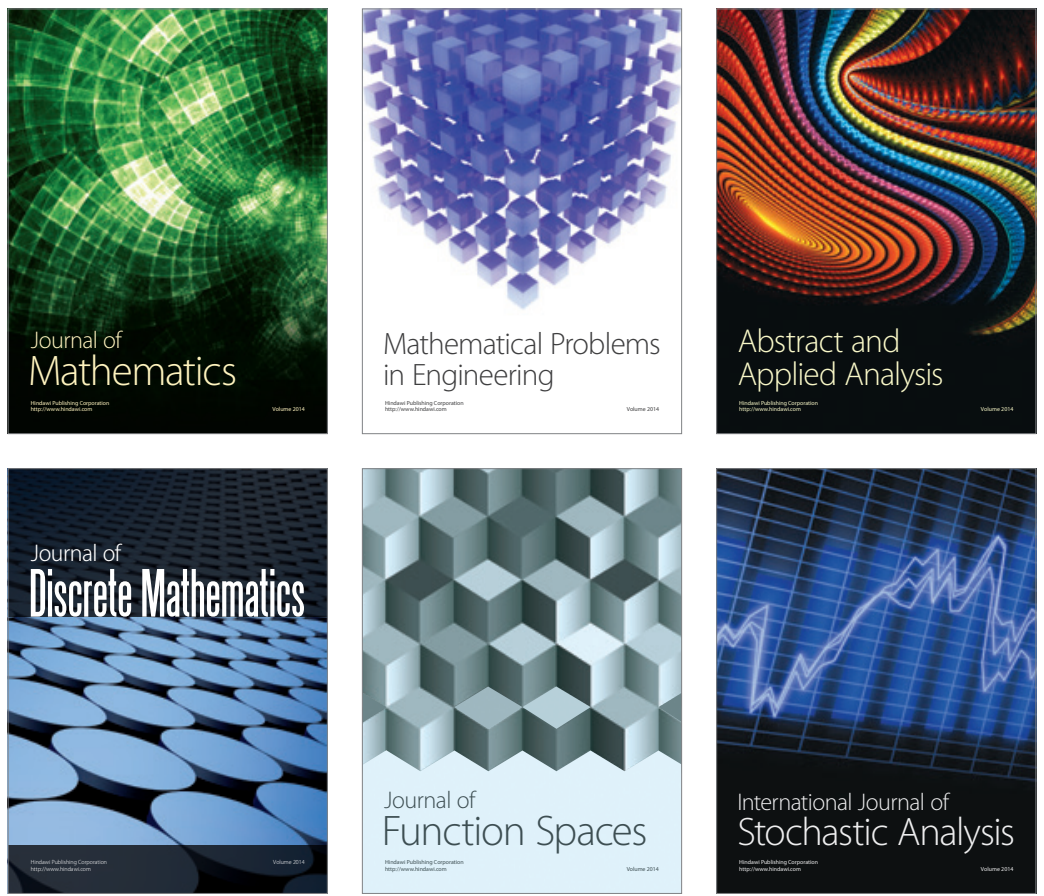

Journal of

Function Spaces

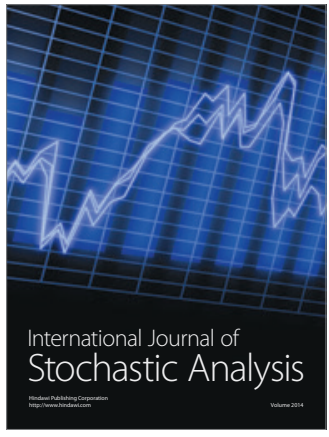

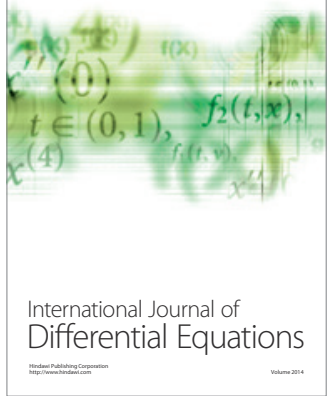
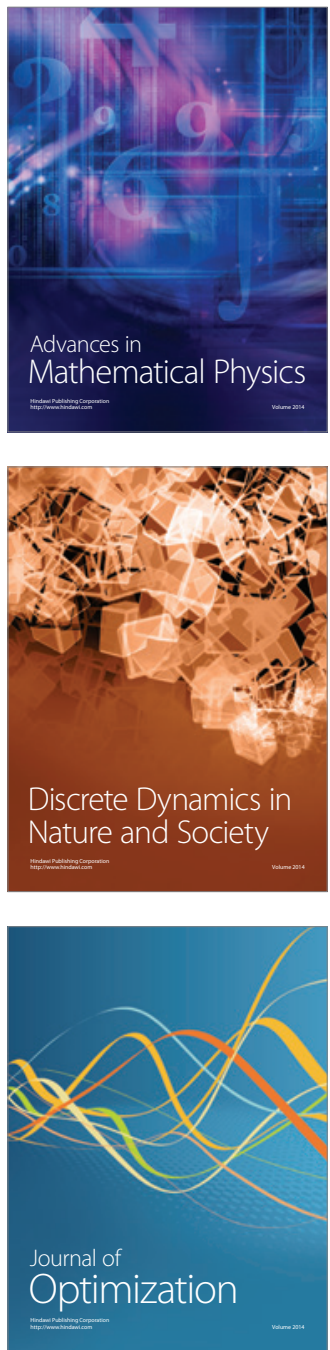